\title{
A REDETERMINATION OF THE CONSTANT OF GRAVITATION
}

\author{
By Paul R. Heyl
}

\section{ABSTRACT}

A redetermination of the constant of gravitation has been made, using the torsion balance in vacuo. The large masses were steel cylinders weighing about $66 \mathrm{~kg}$, and the small masses spheres of gold, platinum, and optical glass. The final result obtained is $6.670 \times 10^{-8} \mathrm{~cm}^{3} \mathrm{~g}^{-1}$ sec. $^{-2}$.

While the results obtained appeared to differ slightly with sinall balls of gold, platinum, or glass, it was shown by a special experiment with the form of torsion balance used by Eötvös that this is not to be attributed to difference in material.

\section{CONTENTS}

Page

1. The nature of the constant of grav

1. The nature of the constant of gravitation ............. 1244

2. Summary of earlier measurements

3. Work of Boys...... 1245

4. Work of Braun

5. Organization of the present work

II. Plan of method

III. Description of apparatus.

1. Observation room

2. The large masses

3. The supporting system $\ldots$

4. The small masses.

5. The moving system

6. The container

7. The optical system

8. Measurements of time

9. Measurements of length

IV. Mathematical theory _..... 1257

1. The attraction of a finite cylinder at any external point...- 1257

2. Formula for time of swing . .

V. Method of observing

1. Centering adjustments

2. Length measurements........... 1277

(a) Horizontal .................. 1277

(b) Vertical

3. Time measurements

VI. Computations............ 1252

VII. Results

1. Gold balls.......

2. Platinum balls

3. Glass balls

4. Mean value

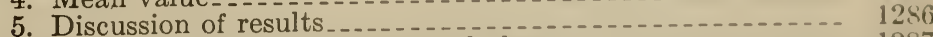




\section{INTRODUCTION}

\section{THE NATURE OF THE CONSTANT OF GRAVITATION}

The constant of gravitation is the multiplying factor $G$ in the formula

$$
F=G \frac{M m}{d^{2}}
$$

giving the force $F$ of gravitational attraction between two masses $M$ and $m$, with a distance $d$ between their centers of attraction.

This constant of nature is one of the few that best deserve the name. It is known to be independent of the nature of the attracting materials to a precision of 6 parts in $10{ }^{1}$ It is known to be independent of the temperature to a precision of about 1 part in $10,000{ }^{2}$ It appears also to be unaffected by the presence of different materials in the space between the attracting bodies. ${ }^{3}$

In theoretical importance the constant of gravitation ranks with the velocity of light and Planck's constant of action. In Einstein's theory of gravitation it appears in a formula for the curvature of space due to the density of distribution of matter. ${ }^{4}$ It undoubtedly has some intimate connection not yet understood with the ultimate structure of the universe.

Practically this constant is of importance as it enables us to determine the mean density of the sun and of the other planets of the solar system.

In thus applying the constant of gravitation it should be pointed out that we are making an assumption-that this constant is the same between the earth and the sun as between two bodies on the earth's surface. The arbitrary nature of this assumption becomes clear when, following Einstein, we regard gravitation as due to space curvature arising from the presence of matter. We are, in fact, assuming space to be everywhere equally resistant to deformation. We do know that the constant of gravitation is approximately the same at several points on the earth's surface, and there is no.reason to suspect the contrary; but we do not know that the same will hold true for different radii, as between the earth and the moon, or even between the center and the surface of the earth.

But if we are wrong in this assumption it is of little practical importance. Our estimates of the masses of the heavenly bodies might be in error, yet their mutual attractions and relative motions would still be the same. However, in the case of a body moving in a very long elliptical orbit, such as a periodic comet, there might be a small differential perturbation from this cause.

And we are not entirely without evidence that our estimate of the mass of the sun, at least, is not grossly erroneous. The Einstein shift of the lines in the solar spectrum depends on the solar mass. While experimental evidence on this point is not of the highest precision, it is perhaps safe to say that our estimate of the mass of the sun is not in error by as much as 20 per cent.

1 Foxtvös, Pekar, and Fekete, Annalen der Physik, 68, 1, pp. 11-66; 1922.
3 P. E. Shaw and N. Davy,

Rusell, 4 strophys. Iavy, Proc. Roy. Soc., 102, pp. 46-47; 1922.

1922; Heyl (summary of J., 54, p. 334; 1921; Eichelberger and Morgan, Astronom. J., No. 795; January,

- Einsteln, Sitzungsberichte der Preuss, Akad, d. Wiss,; Feb, 8, 1917. 


\section{SUMMARY OF EARLIER MEASUREMENTS}

An excellent summary of experimental work on the constant of gravitation may be found in Poynting's article "Gravitation" in the Encyclopædia Britannica (11th ed.). To this is to be added a reference to the work of Crémieu (Comptes rendus, vol. 141, pp. 653, $713 ; 1905$, and vol. 149 , p. $700 ; 1909)$.

The value accepted for this constant for the last 30 years hins rested upon the independent work of Boys and of Braun. ${ }^{5}$ These two experimenters obtained results agreeing to 4 significant figures, $6.658 \times 10^{-8} \mathrm{~cm}^{3} \mathrm{~g}^{-1} \mathrm{sec}^{-2}$, but because their individual values varied in the third figure (by as much as 4 units in Braun's work and by 2 units in that of Boys) Poynting, in his discussion of the subject, adopts the value $6.66 \times 10^{-8}$, subject to an uncertainty of 1 unit in the third figure.

\section{WORK OF BOYS}

Because of the importance of the work of Boys and of Braun it will be well to consider their experiments in more detail. Both used the torsion balance, which has been employed in one form or another by half a dozen different experimenters since the days of Cavendish, but each employed a different arrangement.

Boys, guided by theoretical considerations which show that a short beam may, under certain conditions, give increased sensitivity, constructed his apparatus so that the horizontal distance between centers of the small masses was 1 inch and that between centers of the large masses 6 inches. Since with centers of all masses at the same level the proximity of a large mass to both small ones would largely wipe out the increased sensitivity arising from the short beam, Boys arranged the masses in pairs, one large and one small mass in each pair, the two pairs being at different levels.

The large masses used by Boys weighed about $7.4 \mathrm{~kg}$ each and the small masses each about $2.65 \mathrm{~g}$ :

Boys did not use a vacuum in his apparatus; in fact, he stated in his published paper (p. 70) that he was sure the use of a high vacuum was out of the question. He did, however, carry out one experiment with a hydrogen-filled apparatus, but the results were not encouraging. In fact, no experimenter previous to Braun had made any attempt to work at reduced pressure, and all had experienced trouble from convection currents. Boys was forced to go to great lengths in the way of screens and heat insulation in the attempt to eliminate this difficulty.

Of the two methods of using the torsion balance, direct deflection and time of swing, Boys used the first only. In this method the large masses are set in a position of maximum attraction on the moving system. ( $a$, $a$, fig. 1.) After determining the resting point the moving system then takes up, the large masses are moved to a similar position with opposite turning effect $(b, b)$, and the resting point again noted. Half the angle between the two positions of the moving system measures the deflection produced by the mutual attraction of the masses.

Boys aimed at a precision of 1 part in 10,000, and so designed his apparatus as to afford the accuracy theoretically necessary to uttrin

s C. V. Boys, Phil. Trans. Roy. Soc., A, Pt. 1, p. 1; 1895. Carl Braun, Denkschriften der k. Akad. d. Wiss. (Wien), math. u. naturwiss. Classe, 6t, D. 187; 1897. 
this end. His final results, however, fell considerably short of this precision. Omitting the experiment with the hydrogen-filled apparatus, he obtained the following nine results (omitting a fifth figure given by Boys):

$$
\begin{array}{ll}
6.665 \times 10^{-8} & 6.658 \times 10^{-8} \\
6.670 \times 10^{-8} & 6.653 \times 10^{-8} \\
6.671 \times 10^{-8} & 6.658 \times 10^{-8} \\
6.668 \times 10^{-8} & 6.670 \times 10^{-8} \\
6.655 \times 10^{-8} &
\end{array}
$$

The mean of these values, assumed of equal weight, is 6.663 , with an extreme variation of 0.018 and an average departure from the

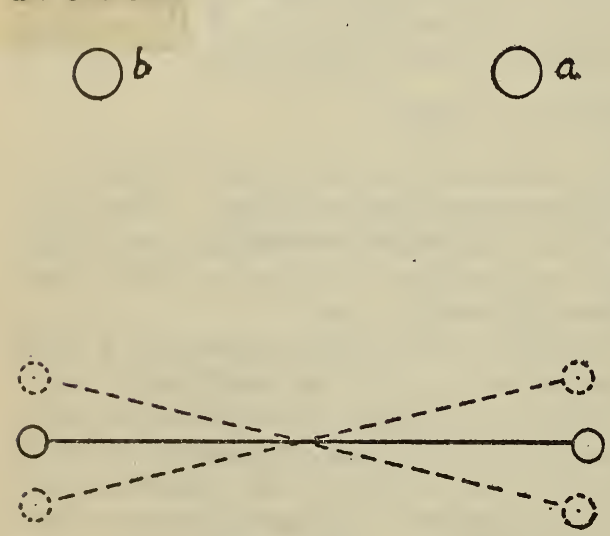
mean of 0.006 . Boys remarks, however, that conditions of disturbance varied greatly in the different experiments, and he selected the fifth, sixth, seventh, and eighth of the above series as being most likely to give a true value. Moreover, he seems to have assigned nearly all the weight to the sixth and eighth, adopting as his final figure $6.658 \times 10^{-8}$.

\section{WORK OF BRAUN}

Braun used a torsion balance of larger dimensions than that constructed by Boys, the distance between centers of the small masses being about $25 \mathrm{~cm}$ and that between centers of the large masses about $42 \mathrm{~cm}$. The large

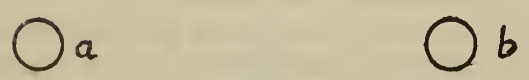

Figure 1.-Direct deflection method masses weighed about $9 \mathrm{~kg}$ each and the small masses each about $54 \mathrm{~g}$. His apparatus was arranged in the usual way, with the centers of all four masses at the same level. For a long beam there is little objection to this plan.

Braun was the first to perform this experiment at reduced pressure, part of his results being obtained at $16 \mathrm{~mm} \mathrm{Hg}$ and part at $4 \mathrm{~mm} \mathrm{Hg}$.

Braun used both the direct deflection and the time-of-swing methods. In the latter method the time of swing is measured with the large masses in two positions which may be called "near" and "far." (Fig. 2.) A little study of this figure will show that in the near position the attraction of the large masses upon the moving system is such as to accelerate the swing, while in the far position it retards it. With the masses and dimensions used by Braun the difference between the two times of swing was about 46 seconds.

Braun stated his results in terms of the mean density of the earth rather than of the constant of gravitation. He published results 
of 46 separate experiments, 26 by the time-of-swing method and 20 by the method of direct deflection, the individual results being weighted from 1 to 4 . The individual results vary by as much as 4 units of the third significant figure.

\section{ORGANIZATION OF THE PRESENT WORK}

So well done was the work of Boys and Braun that the diificulty of improving upon it was clearly recognized. Such a task requires the resources of an institution rather than of an individual. Practically every division in the Bureau of Standards has contributed tangibly or intangibly to the successful completion of this experiment. The work would

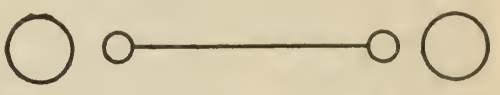
NEAR. probably not have been undertaken had it not been for the encouragement of the director, Dr. George K. Burgess, whose interest in the subject dates back to his student days, and for the like encouragement of Dr. L. J. Briggs, chief of the division of mechanics and sound, whose interest in gravity measurements at sea is well known.

The completion of the undertaking in a reasonable time has been made possible by the cooperation of E. R. Frisby, who has done about 90 per cent of the very laborious computations and, in addition, worked out the formulas for the time of swing.

Drs. H. L. Dryden, of the Bureau of Standards, and E. W. Woolard, of George Washington University, have rendered valuable services in checking the mathematical work at different stages.

The apparatus was constructed almost entirely in the Bureau of Standards instrument shops by J. F. Draper. The task required nearly a year for completion. Acknowledgment is made of the helpful suggestions offered by Mr. Draper in the course of the work.

\section{PLAN OF METHOD}

A study of the work of Boys and Braun led to the conclusion that little was to be hoped for in the way of increased precision by a repetition of the direct-deflection method. As this was carried out by Boys, the only serious objection is the presence of air about the moving system. Yet Boys's work seems to have been very well 
done, for in Braun's experiments this objection does not hold, yet his result by the direct-deflection method confirms that of Boys.

With the time-of-swing method the case is different. Braun says in his published paper that the possibilities of this method much exceed those of the other, and expresses his regret that he was unable to make full use of them. It was therefore decided to use this method only in the repetition of the experiment.

Of the two arrangements of the torsion balance Braun's form seemed preferable to that of Boys for two reasons-it is easier to obtain precision in the important measurement of the horizontal distance between centers of the small masses when this distance is large; and it was planned to increase greatly the size of the large masses, which would have made a very considerable difference of level necessary. The general dimensions of Braun's apparatus, apart from the size of the large masses, seemed ample for obtaining a precision of perhaps 1 part in 10,000. The apparatus was, therefore, designed after the general pattern of that used by Braun, with a considerable increase in the large masses.

Three series of measurements were made, using small masses of gold, platinum, and optical glass.

Photographic recording of the time of swing was considered, and a special form of moving film camera was constructed for this purpose; but it was found that with the distance to be traveled by the beam of light (about $7 \mathrm{~m}$ ) and the passage through 8 pieces of glass with 2 reflections, it was difficult to obtain an image bright enough to be photographed in motion and at the same time sharp enough to be accurate. It was easily possible to obtain a sharp image sufficiently bright for visual observation, and it was therefore decided to make the observations visually, and to record them by a chronograph.

\section{DESCRIPTION OF APPARATUS}

\section{OBSERVATION ROOM}

As an observation room there was available the constant-temperature vault of the bureau, divided into two rooms, one large and one small. This vault is located below the East Building at a depth of about $12 \mathrm{~m}$ below ground. In addition to furnishing uniform temperature this depth eliminated any trouble that might otherwise have arisen from moving masses near by.

The torsion pendulum was set up in the small room, the floor space of which was about $2 \times 2.7 \mathrm{~m}$ and the height about $4 \mathrm{~m}$. The obscrving apparatus was placed in the large room, the intervening brick wall being pierced by a window about $30 \mathrm{~cm}$ square. A pier was built in each room, in the small room at its center, and in the large room close to the far wall, the distance between centers of piers being about $3.5 \mathrm{~m}$. At this distance calculation shows that the effect of the observer's mass upon the moving system is less than one ten-thousandth that of the two large masses. It was deemed advisable, however, that the observer remain in the same place during the whole period of observations, rising from his chair when necessary to attend to the chronograph at his side, but not moving horizontally from his location. 
The observation room proved ideal for the purpose. No vibration was observable, and it was possible to work by day with results equally as good. as those obtained by night. Practically all the work was therefore done by day.

\section{THE LARGE MASSES}

When it is a question of dealing with large masses in an experiment of this kind the nature of the material becomes important. Cavendish (and later Baily) used spheres of lead weighing nearly $160 \mathrm{~kg}$. Such large masses of lead or lead alloy can hardly be trusted to maintain their shape accurately over long periods of time. In addition, there is always the possibility of holes in the casting. To avoid this latter difficulty several experimenters (Braun among the number) have used hollow spheres filled with mercury.

For the present work it seemed most practicable to employ steel, because of ease of accurate shaping and permanence of form. Tool steel of about 0.90 per cent carbon was selected as being most uniform in structure and least liable to segregation. To avoid blowholes and pipes a large ingot of $30 \mathrm{~cm}$ diameter was forged down to $20 \mathrm{~cm}$ and annealed for machining. This work was done at the Washington Navy Yard.

Nearly all previous experimenters have used masses of spherical form because of the simplicity of the calculations involved. The practical difficulties incident to the accurate machining of a sphere of the weight planned (over $60 \mathrm{~kg}$ ), especially in cutting off and rounding the ends, as well as the difficulty of determining accurately the center of the sphere when mounted in place, appeared so great that it was decided to adopt a cylindrical form, thus shifting the burden to the broad shoulders of the mathematician.

After machining the cylinders to size, their dimensions and masses were determined, respectively, by Mr. Miller and Mr. Peffer, of the weights and measures division. These results, in grams and centimeters at $20^{\circ} \mathrm{C}$., are given in Table 1 .

TABLE 1.-Dimensions and masses of cylinders

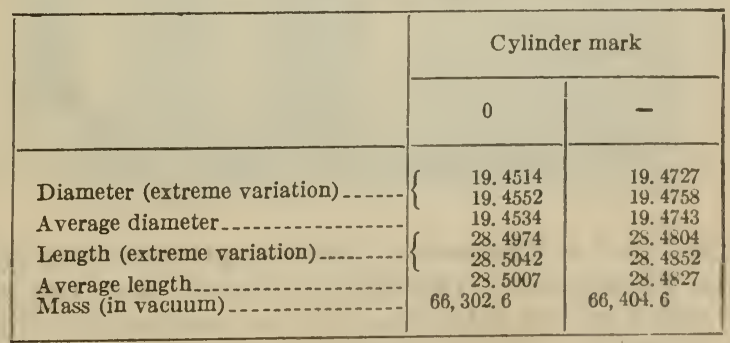

When in place in the apparatus the cylinders are surrounded by air, and the attraction of a cylinder at any point will be that due to the mass of the cylinder in vacuum minus the mass of the displaced air. We may consider a cylinder of this slightly reduced mass superposed upon a continuous and uniform distribution of air. The air being infinite and continuous exerts no resultant attraction at any point within it, and the total resultant attraction is therefore that due to 
the superposed cylinder. Thus the effective values of the mass and density of the cylinder will be found by correcting for displaced air at the temperature, pressure, and humidity existing at the time of the experiment.

Calculation shows that for a variation in relative humidity from 57 to 86 and for the usual pressure variation the mass of air displaced by a cylinder is constant to the first decimal place, namely, $9.9 \mathrm{~g}$; and this is also the precision to which the masses of the cylinders are known. Applying this correction to the values given in Table 1 we obtain the effective values given in Table 2:

TABLE 2.-Effective masses and densities of cylinders

\begin{tabular}{|c|c|c|}
\hline & \multicolumn{2}{|c|}{ Cylinder mark } \\
\hline & 0 & - \\
\hline 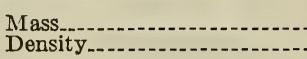 & $\begin{array}{l}66,292.7 \\
7.82583\end{array}$ & $\begin{array}{l}66,394.7 \\
7.82608\end{array}$ \\
\hline
\end{tabular}

Uniformity of density is an important consideration. There can hardly be any question of appreciable pipes or blowholes after the preliminary reduction by forging, but we must consider the possibility that by this forging the outer layers of the cylinder may havebeen made slightly denser than the center. Segregation also might cause a change in density of like distribution. As a check upon this, samples were taken from the bosses cut off from an end of each cylinder in the final stages of machining, and the density of these samples determined. Each sample had a diameter of about one-fifth that of the cylinder, and may thus be taken as representing approximately the density of the cylinder near its axis. Table 3 shows the comparison of these densities with the calculated values for the whole cylinders.

\section{TABLE 3.-Variation in density of cylinders (in vacuum)}

\begin{tabular}{|c|c|c|}
\hline & \multicolumn{2}{|c|}{ Cylinder mark } \\
\hline & 0 & - \\
\hline $\begin{array}{l}\text { Density near axis } \\
\text { Calculated mean density... }\end{array}$ & $\begin{array}{l}\text { 7. } 8255 \\
\text { 7. } 8270\end{array}$ & $\begin{array}{l}7.8250 \\
7.8273\end{array}$ \\
\hline
\end{tabular}

In no case is there a difference in density greater than about 1 part in 4,000 . An approximate calculation shows that the error introduced in the value of the attraction by the assumption (for instance) of a uniform density of 7.8273 instead of one varying uniformly from 7.8250 at the center to 7.8285 at the outer surface (with a mean of 7.8273 ) amounts to less than 1 part in 50,000, negligible for present purposes.

\section{SUPPORTING SYSTEM}

The large masses were supported so as to be free to turn in azimuth about a vertical axis midway between them. The general arrangement is shown in Figure 3. 
B. S. Journal of Research, RP256

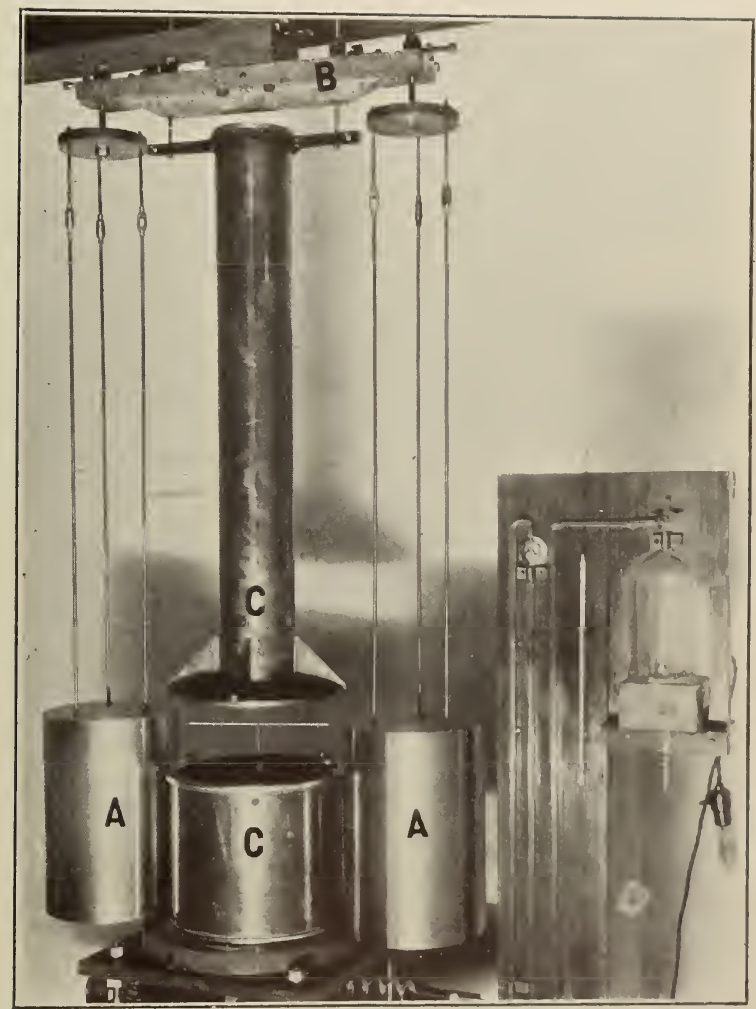

FraURE 3.-General arrangement of apparatus 
Two $15 \mathrm{~cm}$ I beams were set into the brick walls of the room with a space of about $20 \mathrm{~cm}$ between them. Across these beams was fastened by screw clamps a steel plate $2.5 \mathrm{~cm}$ thick and $15 \mathrm{~cm}$ wide. This crosspiece was pierced at the center by a hole $3.7 \mathrm{~cm}$ in diameter, through which passed by a snug fit the vertical axis of the supporting system.

This vertical axis was capped at the upper end by a disk resting upon a ring of steel balls rolling in a channel cut in the crosspiece. Fastened to the upper surface of the desk was a graduated circle 35 $\mathrm{cm}$ in diameter provided with a vernier reading to $0.1^{\circ}$.

The vertical axis carried at its lower end an arrangement of dovetailed blocks allowing two sliding adjustments at right angles, for the purpose of centering the cylinders with respect to the point of support of the pendulum.

Fastened to the lowest of these sliding blocks was the crossbeam $B$ (fig. 3 ), from the ends of which hung the cylinders $A, A$. Each cylinder was supported by three steel rods, $0.5 \mathrm{~cm}$ in diameter, screwed into the cylinder by tapped holes, each hole threaded all the way down to a flat bottom against which the flat end of the rod fitted. Each rod was provided with a lock nut where it entered the cylinder and, near its upper end, with a turnbuckle by which the cylinder could be leveled. The vertical adjustment of the cylinder as a whole could be secured by raising or lowering a disk from which the three rods hung. Each disk was supported by a bolt and nut passing through a slot in the crossbeam $B$, thus pernitting separate horizontal adjustments of the cylinders.

\section{THE SMALL MASSES}

Small masses, spherical in shape, were used of three different materials, gold, platinum, and optical glass. In each case the mass was about $50 \mathrm{~g}$.

This use of different materials was not prompted by any suspicion that a specific difference in attraction might be found. Rather was it the result of circumstances. The use of gold balls was suggested by the employment of this metal by Boys and by the fact that Braun used gilded balls of brass. There was no objection to this in the case of the work of Boys, who did not employ a vacuum, and Braun mentions no trouble with his gilded balls; but in the present work it was found on opening the container, after a period of several months required for the observations, that the gold balls had absorbed quite appreciable quantities of mercury, probably derived in vapor form from the manometer connected to the container.

A second set of measurements was made with platinum balls, coated thinly with lacquer by dipping. No appreciable change of weight was observed in this case.

A third set was made with balls of optical glass, the idea being that holes in the interior could be detected visually.

The metallic balls were constructed from ingots which (in the case of gold) had been fused in a vacuum furnace to avoid gas bubbles. The platinum ingots were fused in the oxyhydrogen flame in the usual manner. The ingots were first roughly hammered into shape between hemispherical steel dies, as described by Boys, and finally $18296^{\circ}-30-5$ 
shaped to size by a scraping process suggested by J. F. Draper, of the hureau's instrument shops.

In a piece of thin sheet steel was cut a circular hole of the diameter desired for the finished sphere. The ball, after having been hammered to a diameter but little in excess of the final size, was laid in this hole and turned this way and that between the thumb and finger until it had scraped its way through the hole. In the case of the platinum balls this operation was conducted under water. The resulting balls were so nearly spherical that micrometer measurements (to about $2.5 \mu$ ) could detect no variation in diameter.

In each metallic ball there was drilled a hole $1 \mathrm{~mm}$ in diameter and $3 \mathrm{~mm}$ deep, which was threaded. A piece of copper wire $6 \mathrm{~mm}$ long, threaded for half its length, was screwed in to the hole. The projecting portion of the wire was shaped into a hook.

The glass balls were drilled with holes $1 \mathrm{~mm}$ in diameter and $4 \mathrm{~mm}$ deep, the lower part of the whole being enlarged to a diameter of 2

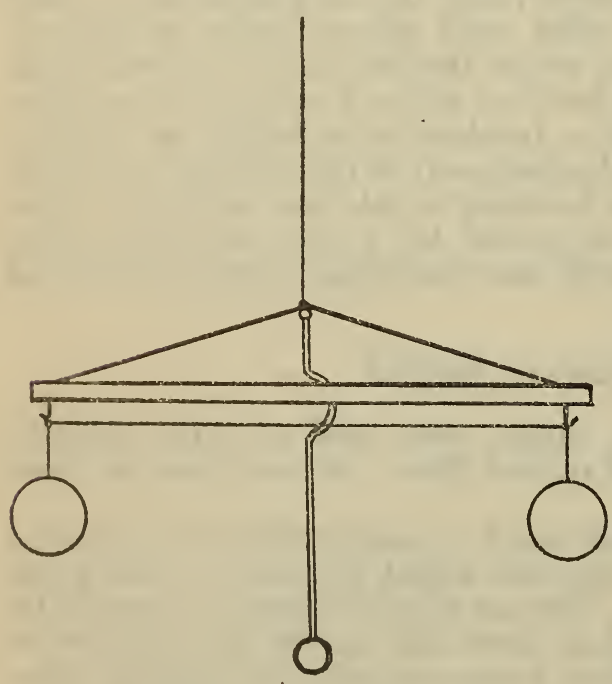

FIGURE 4.-The moving system $\mathrm{mm}$ by a dental burr. In this hole was inserted a doubled piece of copper wire, loop downward. The space between wire and glass was packed with small scraps of tin foil after the manner of filling a tooth, using a blunted needle and light taps from a small hammer. The tinfoil packing was stopped about half a millimeter from the top of the hole and the rest of the space filled with wax. The protruding ends of the wire were joined by a trace of solder and bent into a hook.

In each case the mass of a ball was determined before drilling it. At the conclusion of a set of measurements the ball was cut from the suspending filament close to the hook, and reweighed with hook, knot of filament, and trace of wax used to keep the knot from slipping. The slight excess of mass over that of the solid ball was regarded for purposes of calculation as a massive point added at the north pole of the ball.

\section{THE MOVING SYSTEM}

The moving system, or torsion pendulum, consisted of the two small masses, a light separating rod and the necessary supporting filaments. (Fig. 4.) In the design of this system the object was to throw as much as possible of the moment of inertia into the balls so that the system should approximate a simple pendulum. Actually over 99 per cent of the moment of inertia was thus concentrated.

The horizontal separating rod was of aluminum, $0.24 \mathrm{~cm}$ in diameter, $20.59 \mathrm{~cm}$ long, and weighted in vacuum $2.4401 \mathrm{~g}$. Close to each end 
was drilled a small hole through which passed the ends of a copper wire constituting a truss. These ends, after passing through the rod, were bent into hooks. The cooper truss wire was $0.36 \mathrm{~mm}$ in diameter, $22.1 \mathrm{~cm}$ long and weighed in racuum $0.2385 \mathrm{~g}$.

The balls were hung from a piece of tungsten lamp filament, 0.025 $\mathrm{mm}$ in diameter, tied to their hooks, and laid across the hooks at the ends of the copper truss wire. In tying the filament to the hooks of the balls the filament was wrapped four or five times around the hook and the end twisted around the main portion of the filament. This twist was held from slipping by a trace of wax. In cutting off the balls for the final weighing the twisted portion of the filament was included.

The supporting filament for the moving system as a whole was also of tungsten, $0.025 \mathrm{~mm}$ in diameter, commercial lamp filament annealed in hydrogen at the factory. Tungsten is preferable to silica in that it may be confidently relied on for a long program of observations. Filaments of silica, as Boys found, occasionally break for no apparent reason, often with loss of previous observations. Moreover, tungsten may be tied firmly to the object which it supports.

Our experience indicates that tungsten is quite reliable, returning well to its resting point after large deflections. One such filament was in use three years, during which the apparatus experienced a slight earthquake.

The supporting filament was about $1 \mathrm{~m}$ long, provided with copper wire hooks at its ends. By the lower hook the copper truss wire hung at its apex and was fastened by a trace of wax. The upper hook was fastened to a morable torsion head contained in the chimney of the bell jar. This device (patterned after one used by Braun) consisted of a train of wheel work, to the slow motion end of which the upper hook of the supporting filament was attached. By turning the other end of the train a rery small twist could be given to the filament to correct, if necessary, for drift of the resting point. In order to operate this device from outside without breaking the vacuum a bar magnet was attached to the upper end of the train. By the use of a large horseshoe magnet outside the chimney the requisite twist could be given.

The train of wheel work was geared to a ratio of $3,600: 1$, so that one complete turn of the upper end produced a twist of one-tenth of a degree in the filament.

The moving system was provided with a silvered glass mirror, about. $2 \mathrm{~cm}$ in diameter, $1 \mathrm{~mm}$ thick, weighing about $0.6 \mathrm{~g}$, ground and polished optically flat. This mirror was attached at its upper edge by a little wax to a brass rod $1 \mathrm{~mm}$ in diameter. At its upper end this brass rod was provided with a small wire loop by which it was hung from the lower hook of the main suspension filament. The brass rod, where it passed the horizontal aluminum rod, was bent into a detour to avoid contact. (Fig. 4.)

The total mass of the swinging system was about $102 \mathrm{~g}$. The breaking strength of the suspending filament was about $165 \mathrm{~g}$.

To start the moving system gravitational attraction was employed. 'Two bottles, each holding about $2 \mathrm{~kg}$ of mercury, were placed iluse to the container in positions of maximum attraction for the penclulum. When the latter had been twisted to its greatest extent (which required about 15 minutes) the bottles were placed so as to reverse the attrac- 
tion. By repeatedly reversing the positions of the mercury bottles in time with the swing of the pendulum the latter could be given any amplitude desired. In two hours time an amplitude of about $4^{\circ}$ could be obtained, which (at the reduced pressure employed) was sufficient to keep the pendulum swinging for 20 hours.

\section{THE CONTAINER}

The container was of metal resting on a plate-glass base. The general shape is shown in Figure 3 . The tall chimney was of brass, open at the upper end, which was closed by a piece of plate glass. This permitted observation of the magnet connected with the wheel work. The wide lower portion of the container and the lid fitting it were, in the series of measurements with the gold balls, of brass wrapped with thin sheets of silicon steel for magnetic shielding. This arrangement proved cumbersome, and in the measurements with platinum and glass balls this part of the container was made of a piece of wroughtiron tubing with a lid of the same material. A circular piece of iron was laid inside the container upon the plate-glass base, thus completing the magnetic shielding.

This precuation was necessary because of the steel cylinders, which when moved from the near to the far position altered the earth's magnetic field in the vicinity of the pendulum. Since all materials are either paramagnetic or diamagnetic to an appreciable degree, and since the gravitational attraction of a cylinder upon the nearest ball was only about 0.001 dyne, it is quite possible that the effect of the change in magnetic field upon the time of swing of the moving system might be comparable with that of gravitational attraction.

This suspicion was experimentally confirmed in the case of the brass container used with the gold balls. Before applying the silicon steel wrapping a bar electromagnet was mounted outside the container with one pole in the maximum attraction position with respect to one of the balls. On energizing the magnet the ball was repelled with an acceleration approximately equal to that produced by one of the mercury bottles. By raising the magnet to the level of the aluminum rod an attraction was produced. The magnetic susceptibility of gold is -0.15 and that of aluminum +0.65 .

By wrapping the lower part of the container with several layers of thin silicon steel sheet these disturbances were completely prevented. In the later form of the apparatus, used with platinum and glass balls, the wrought-iron wall of the container was similarly protective.

To test the shielding more closely, measurements of time of swing were made with the outside magnet alternately magnetized and demagnetized. The magnetic field at the outer surface of the container with the electromagnet excited was about twelve times as strong as the earth's horizontal ficld, as determined by the rate of vibration of a small compass needle. The strength of field close to one of the large steel cylinders was about three times that of the earth's field, or onefourth that used for testing the shiclding, and the presence or absence of the testing field produced no effect upon the time of swing as great as 1 part in 10,000. Any effect to be expected by moving the cylinders must therefore be less than 1 part in 40,000 .

A pressure of about $2 \mathrm{~mm}$ of mercury was maintained in the container, the joints of which were sealed by soft universal wax. A mercury barometric seal was used instead of a stopcock. 
It may be of interest to record here the effect of pressure upon the damping exhibited by the moving system. Table 4 gives the damping factor (ratio of successive amplitudes in the same direction) at various pressures.

TABLE 4.

\begin{tabular}{|c|c|}
\hline $\begin{array}{c}\text { Damping } \\
\text { factor }\end{array}$ & $\begin{array}{c}\text { Pressure, } \\
\text { mm mer- } \\
\text { cury }\end{array}$ \\
\cline { 1 - 2 } 0.70 & 150 \\
.84 & 50 \\
.85 & 20 \\
.85 & 10 \\
.85 & 7 \\
.85 & 5 \\
.86 & 3 \\
.87 & 1.5 \\
\hline
\end{tabular}

Windows were provided at strategical points in the container for the purpose of obtaining such measurements as the level of the centers of the balls and the position of the filament supporting the moving system.

When it was desired to raise the chimney and lid of the container a yoke was clamped about the upper end of the chimney. From the ends of this yoke two threaded rods passed up through holes in the beam $B$ (fig. 3) and were supported above by nuts. By turning these nuts the upper portion of the container and the whole moving system could be raised or lowered slowly and steadily enough to avoid breaking the suspensiun.

The temperature of the container was read by means of a thermometer fastened closely in parallel contact with the chimney, the bulb touching the metal. On its outer side the bulb was shielded by a layer of cotton, the whole being bound to the chimney by strips of adhesive tape. The temperature inside the container was assumed to be that of the metal case as shown by the thermometer.

\section{THE OPTICAL SYSTEM}

The basis of reference for observing the time of swing was a scale of millimeters in the form of a photographic negative on glass, bright lines on a dark background. (Fig. 5.) Parallel to the scale and close to it was a broad bright band against which could be seen the vertical cross wire of the observing telescope. The scale was mounted in a metal box and illuminated from behind by several small electric lamps, and was

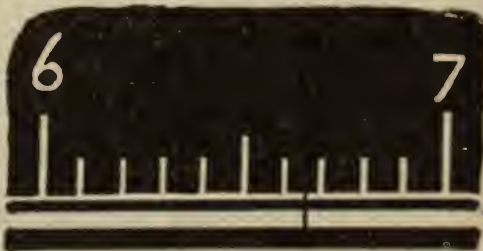

Figure 5.-Scale and cross wire set on a pier in the observing room, $3.5 \mathrm{~m}$ from the torsion apparatus.

The light from the scale passed to a reflecting prism below the glass base of the container, which reflected it vertically upward, passing through the iron floor of the container by a hole. The light then encountered a second prism which reflected it horizontally to the mirror attached to the moving system. The reflected beam retraced 
approximately its entering path and was finally received by a telescope on the observing pier, $3.5 \mathrm{~m}$ distant from the center of the moving system. A motion of one minute of arc of the moving system produced a shift of about $2 \mathrm{~mm}$ of the scale in the field of the telescope.

\section{MEASUREMENT OF TIME}

The working standard of time was a Riefler clock set up in a constant-temperature vault in another building of the bureau, and rated daily against the Naval Observatory signals. Its usual daily rate was but a few hundredths of a second. Second signals from this clock are electrically transmitted to all parts of the bureau laboratories.

The transits of the pendulum were recorded on a 2-pen chronograph. One pen recorded the clock signals and the other the transits of the scale divisions as observed by the operator.

In making the observations it was customary to adjust the resting point of the pendulum by means of the torsion head to somewhere between the twelfth and thirteenth centimeter marks on the scale, and to record the transit of every millimeter from the eleventh to the fourteenth centimeter. The calculation of the time of swing will be described in a later section.

\section{MEASUREMENTS OF LENGTH}

The length measurements fall into two classes-those in which approximate values are sufficient and those requiring higher precision. As an example oi the first class may be mentioned the vertical difference of level between centers of balls and cylinders. In the second class are found the distance between centers of balls and the distance between cylinders.

Measurements of the first class were made to $0.1 \mathrm{~mm}$, mostly with a kathetometer. Measurements of the second class were made with an optical compass similar to that used by Boys; it consisted essentially of two micrometer microscopes mounted approximately parallel to each other on a rigid bar. The distance between microscopes could be adjusted as desired.

A supporting framework of iron was clamped to the pier carrying the torsion pendulum, and by leveling the optical compass upon this framework the microscopes could be directed to view any two points whose horizontal distance apart might be desired. When the setting of the microscopes had been made the optical compass was placed in another stand so as to view a scale below it.

This scale was made from a steel bar $2.5 \mathrm{~cm}$ square in cross section, provided with several platinum-faced plugs, upon which fine graduations were traced. Sufficient plugs were provided to give the various lengths required. The scale was calibrated in terms of the standard ineter by Doctor Judson, of the weights and measures division of the bureau.

Steel was used as a basis for the scale rather than invar, as the (xperience of the bureau has been that invar, while low in expansion, shows much hysteresis, and is on the whole less satisfactory for such is purpose than steel. The coefficient of steel may be higher, but it is more safely to be counted upon than that of invar. 
The steel chosen for this purpose was machined to size and allowed to age for several months to eliminate tho warping that usually follows removal of the surface layers. This process of aging was accelerated by rattling the bar in a shaking apparatus for several periods of $\Omega$ day each. The finished scale was mounted in a wooden case provided

\section{MATHEMATICAL THEORY}

1. THE ATTRACTION OF A FINITE CYLINDER AT ANY EXTERNAL

The use in the Cavendish experiment of attracting bodies of other than a spherical shape has been rare. Boys in one of his experiments

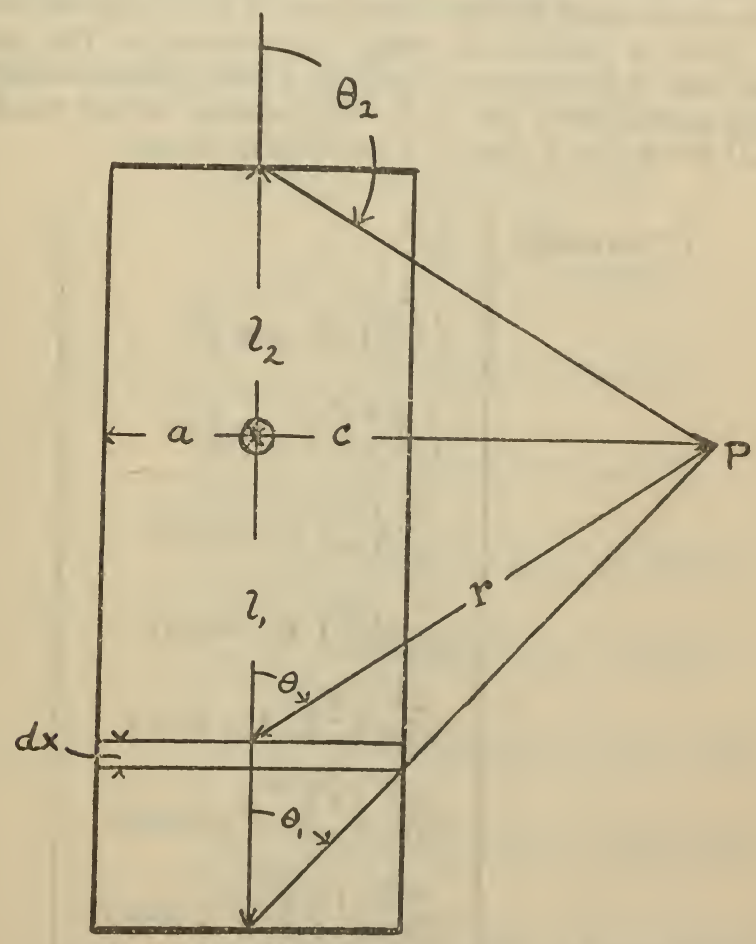

FIGURE 6.-Attraction of finite cylinder at outside point

used cylinders of gold one-fourth inch long and the same in diameter. He states that calculation showed that the attraction of such a cylinder at a point at the center of one of the large balls was less than that which would have been exerted had the cylinder been a sphere of equal mass by 3 parts in 10,000. Small masses of cylindrical and also of chain form were used by P. E. Shaw ${ }^{6}$ in his work on a supposed temperature coefficient of gravitation, but as his work was concerned with relative rather than absolute values it was not necessary to calculate the actual force. Crémieu used large cylindrical masses, but does not mention the computations.

P. E. Shaw, Phil. Trans. Roy. Soc., A, 216, 1916. 
Calculations for nonspherical masses are not simple; in fact, the labor involved in making a nonspherical calculation to anything but a rough approximation has rather been looked upon as prohibitive. For practical reasons earlier mentioned it was desirable to use large masses of cylindrical form. It was an agreeable surprise to find that in the case of the dimensions used it was possible to obtain a formula in zonal harmonics which converged fairly rapidly. Working to 1 part in 100,000 there were necessary only seven terms in the near position and six in the far position.

A formula for the attraction of a finite cylinder at any external point has never, as far as we know, been published. It may therefore be a convenience to future workers if the development of this formula is given somewhat in detail. The best method of procedure is to integrate the expression for the attraction of a circular disk. (Fig. 6.)

Byerly ${ }^{7}$ gives a formula in zonal harmonics for the potential $V$ of a circular disk of thickness $d x$, density $\rho$ and radius $a$ at any external point whose coordinates are $r, \theta$, the formula being valid if $r>a$. Formula (2) gives this value of $V$ to eleven terms.

$$
V=2 \pi \rho a G d x\left\{\begin{array}{l}
+\frac{1}{2} \frac{a}{r} \\
-\frac{1}{8}\left(\frac{a}{r}\right)^{3} P_{2}(\cos \theta) \\
+\frac{1}{16}\left(\frac{a}{r}\right)^{5} P_{4}(\cos \theta) \\
-\frac{5}{128}\left(\frac{a}{r}\right)^{7} P_{6}(\cos \theta) \\
+\frac{7}{256}\left(\frac{a}{r}\right)^{9} P_{8}(\cos \theta) \\
-\frac{21}{1024}\left(\frac{a}{r}\right)^{11} P_{10}(\cos \theta \\
+\frac{33}{2048}\left(\frac{a}{r}\right)^{13} P_{12}(\cos \theta) \\
-\frac{429}{32768}\left(\frac{a}{r}\right)^{15} P_{14}(\cos \theta) \\
+\frac{715}{65536}\left(\frac{a}{r}\right)^{17} P_{16}(\cos \theta) \\
-\frac{2431}{262144}\left(\frac{a}{r}\right)^{19} P_{18}(\cos \theta) \\
+\frac{4199}{524288}\left(\frac{a}{r}\right)^{21} P_{20}(\cos \theta)
\end{array}\right.
$$

\footnotetext{
'Byerly, Fourier's Series, p. 155, art. 80.
} 
Following the notation of Figure 6 in which $x$ is measured positively downward from 0 , and expressing all variables in (2) in terms of $\theta$,

$$
\left.\begin{array}{rl}
\mathrm{n} \theta & =\frac{c}{r} \\
\frac{a}{r} & =\frac{a}{c} \sin \theta \\
x & =c \cot \theta \\
d x & =-\frac{c d \theta}{\sin ^{2} \theta}
\end{array}\right\}
$$

and integrating for $\theta$ from $\theta_{1}$ to $\theta_{2}$ we obtain:

$$
V=-2 \pi \rho a c G\left|\begin{array}{l}
+\frac{1}{2} \frac{a}{c} \int \frac{d \theta}{\sin \theta} \\
-\frac{1}{8}\left(\frac{a}{c}\right)^{3} \int \sin \theta \cdot P_{2}(\cos \theta) d \theta \\
+\frac{1}{16}\left(\frac{a}{c}\right)^{5} \int \sin ^{3} \theta \cdot P_{4}(\cos \theta) d \theta \\
-\frac{5}{128}\left(\frac{a}{c}\right)^{7} \int \sin ^{5} \theta \cdot P_{6}(\cos \theta) d \theta \\
+\frac{7}{256}\left(\frac{a}{c}\right)^{9} \int \sin ^{7} \theta \cdot P_{8}(\cos \theta) d \theta \\
-\frac{21}{1024}\left(\frac{a}{c}\right)^{11} \int \sin ^{9} \theta \cdot P_{10}(\cos \theta) d \theta \\
+\frac{33}{2048}\left(\frac{a}{c}\right)^{13} \int \sin ^{11} \theta \cdot P_{12}(\cos \theta) d \theta \\
-\frac{429}{32768}\left(\frac{a}{c}\right)^{15} \int \sin ^{13} \theta \cdot P_{14}(\cos \theta) d \theta \\
+\frac{715}{65536}\left(\frac{a}{c}\right)^{17} \int \sin ^{15} \theta \cdot P_{16}(\cos \theta) d \theta \\
-\frac{2431}{262144}\left(\frac{a}{c}\right)^{19} \int \sin ^{17} \theta \cdot P_{18}(\cos \theta) d \theta \\
+\frac{4199}{524288}\left(\frac{a}{c}\right)^{21} \int \sin ^{19} \theta \cdot P_{20}(\cos \theta) d \theta
\end{array}\right|
$$


Byerly (p. 151) gives expansions of $P_{m}(x)$ up to $m=8$. Additional values follow:

$$
\begin{aligned}
& P_{10}(x)=\frac{1}{256}\left(\begin{array}{l}
\left(46189 x^{10}-109395 x^{8}+90090 x^{6}\right. \\
\left.-30030 x^{4}+3465 x^{2}-63\right) \\
P_{12}(x)=\frac{1}{1024} \\
P_{14}(x)=\frac{1}{2048} \\
P_{16}(x)=\frac{1}{32768} \\
-1021020 x^{12}-1939938 x^{10}+2078505 x^{8} \\
\left(5014575 x^{14}-16900975 x^{12}+22309287 x^{10}\right. \\
-14549535 x^{8}+4849845 x^{6}-765765 x^{4} \\
\left.+45045 x^{2}-429\right) \\
\left(300540195 x^{16}-1163381400 x^{14}\right. \\
+1825305300 x^{12}-1487285800 x^{10} \\
+669278610 x^{8}-162954792 x^{6} \\
\left.+19399380 x^{4}-875160 x^{2}+6435\right) \\
P_{18}(x)=\frac{1}{65536} \\
\left(2268783825 x^{18}-9917826435 x^{16}\right. \\
+18032411700 x^{14}-17644617900 x^{12} \\
+10039179150 x^{10}-3346393050 x^{8} \\
+624660036 x^{6}-58198140 x^{4} \\
\left.+2078505 x^{2}-12155\right) \\
\\
\left(34461632205 x^{20}-167890003050 x^{18}\right. \\
+347123925225 x^{16}-396713057400 x^{14} \\
+273491577450 x^{12}-116454478140 x^{10} \\
+30117537450 x^{8}-4461857400 x^{6} \\
\left.+334639305 x^{4}-9699690 x^{2}+46189\right)
\end{array}\right.
\end{aligned}
$$

Making the necessary substitutions in formula (4) we obtain:

$$
V=-2 \pi \rho a c G \mid \begin{aligned}
& +\frac{1}{2} \frac{a}{c} \int \frac{d \theta}{\sin \theta} \\
& -\frac{1}{16}\left(\frac{a}{c}\right)^{3} \int \sin \theta\left(3 \cos ^{2} \theta-1\right) d \theta \\
& +\frac{1}{12 \delta}\left(\frac{a}{c}\right)^{5} \int \sin ^{3} \theta \cdot\left(35 \cos ^{4} \theta-30 \cos ^{2} \theta+3\right) d \theta \\
& -\frac{5}{2048}\left(\frac{a}{c}\right)^{7} \int \sin ^{5} \theta\left(231 \cos ^{6} \theta-315 \cos ^{4} \theta\right. \\
& \left.+105 \cos ^{2} \theta-5\right) d \theta
\end{aligned}
$$


$V=-2 \pi \rho a c G \mid+\frac{7}{32768}\left(\frac{a}{c}\right)^{9} \int \sin ^{7} \theta\left(6435 \cos ^{2} \theta-12012 \cos ^{4} \theta\right.$

$+6930 \cos ^{4} \theta-1260 \cos ^{2} \theta$

$+35) d \theta$

$-\frac{21}{262144}\left(\frac{a}{c}\right)^{11} \int \sin ^{9} \theta\left(46189 \cos ^{10} \theta\right.$

$-109395 \cos ^{8} \theta$

$+90090 \cos ^{6} \theta-30030 \cos ^{4} \theta$

$\left.+3465 \cos ^{2} \theta-63\right) d \theta$

$+\frac{33}{2097152}\left(\frac{a}{c}\right)^{13} \int \sin ^{11} \theta\left(676039 \cos ^{12} \theta\right.$

$-1939938 \cos ^{10} \theta$

$+2078505 \cos ^{8} \theta$

$-1021020 \cos ^{6} \theta$

$+225225 \cos ^{4} \theta$

$\left.-18018 \cos ^{2} \theta+231\right)(1 \theta$

$-\frac{429}{67108864}\left(\frac{a}{c}\right)^{15} \int \sin ^{13} \theta\left(5014575 \cos ^{14} \theta\right.$

$-16900975 \cos ^{12} \theta$

$+22309287 \cos ^{10} \theta$

$-14549535 \cos ^{8} \theta$

$+4849845 \cos ^{6} \theta$

$-765765 \cos ^{4} \theta$

$\left.+45045 \cos ^{2} \theta-429\right) d \theta$

$+\frac{715}{2147483648}\left(\frac{a}{c}\right)^{17} \int \sin ^{15} \theta\left(300540195 \cos ^{16} \theta\right.$

$-1163381400 \cos ^{14} \theta$

$+1825305300 \cos ^{12} \theta$

$-1487285800 \cos ^{10} \theta$

$+669278610 \cos ^{8} \theta$

$-162954792 \cos ^{6} \theta$

$+19399380 \cos ^{4} \theta$

$-875160 \cos ^{2} \theta$

$+6435) d \theta$

$-\frac{2431}{17179869184}\left(\frac{a}{c}\right)^{19} \int \sin ^{17} \theta\left(2268783825 \cos ^{18} \theta\right.$

$-9917826435 \cos ^{16} \theta$

$+18032411700 \cos ^{14} \theta$

$-17644617900 \cos ^{12} \theta$

$+10039179150 \cos ^{10} \theta$

$-3346393050 \cos ^{8} \theta$

$+624660036 \cos ^{6} \theta$

$-58198140 \cos ^{4} \theta$

$+2078505 \cos ^{2} \theta$

$-12155) d \theta$

(6) 


$$
\begin{aligned}
& V=-2 \pi \rho a c G\left(+\frac{4199}{137438953472}\left(\frac{a}{c}\right)^{21} \int \begin{array}{c}
\sin ^{19} \theta(34461632205 \\
\cos ^{20} \theta
\end{array}\right. \\
& \text { - } 167890003050 \\
& \cos ^{18} \theta \\
& +347123925225 \\
& \cos ^{16} \theta \\
& -396713057400 \\
& \cos ^{11} \theta \\
& +273491577450 \\
& \cos ^{12} \theta \\
& -116454478140 \\
& \cos ^{10} \theta \\
& +30017537450 \cos ^{8} \theta \\
& -4461857400 \cos ^{6} \theta \\
& +334639305 \cos ^{4} \theta \\
& -9699690 \cos ^{2} \theta \\
& +46189) d \theta
\end{aligned}
$$

Integrating and applying the limits $\theta_{1}$ and $\theta_{2}$

$$
\begin{aligned}
& V=-2 \pi \rho a c G\left(+\frac{1}{2} \frac{a}{c}\left[\log \tan \frac{\theta_{2}}{2}-\log \tan \frac{\theta_{1}}{2}\right]\right. \\
& -\frac{1}{16}\left(\frac{a}{c}\right)^{3}\left[\cos \theta_{2} \sin ^{2} \theta_{2}-\cos \theta_{1} \sin ^{2} \theta_{1}\right] \\
& +\frac{1}{128}\left(\frac{a}{c}\right)^{5}\left[\begin{array}{lllllll}
5 & \cos ^{3} \theta_{2} & \sin ^{4} & \theta_{2}-3 & \cos \theta_{2} & \sin ^{4} \theta_{2}
\end{array}\right. \\
& \left.+ \text { similar terms of opposite sign in } \theta_{1}\right] \\
& -\frac{5}{2048}\left(\frac{a}{c}\right)^{7}\left[21 \cos ^{5} \theta_{2} \sin ^{6} \theta_{2}-\frac{70}{3} \cos ^{3} \theta_{2} \sin ^{6} \theta_{2}\right. \\
& \left.+5 \cos \theta_{2} \sin ^{6} \theta_{2}+\text { etc. }\right] \\
& +\frac{7}{32768}\left(\frac{a}{c}\right)^{9}\left[429 \cos ^{7} \theta_{2} \sin ^{8} \theta_{2}-693 \cos ^{5} \theta_{2} \sin ^{8} \theta_{2}\right. \\
& +315 \cos ^{3} \theta_{2} \sin ^{8} \theta_{2}-35 \cos \theta_{2} \sin ^{8} \theta_{2}+\text { etc. } \\
& -\frac{21}{262144}\left(\frac{a}{c}\right)^{11}\left[2431 \cos ^{9} \theta_{2} \sin ^{10} \theta_{2}\right. \\
& -5148 \cos ^{7} \theta_{2} \sin ^{10} \theta_{2}+\frac{18018}{5} \cos ^{5} \theta_{2} \sin ^{10} \theta_{2} \\
& -924 \cos ^{3} \theta_{2} \sin ^{10} \theta_{2}+63 \cos \theta_{2} \sin ^{10} \theta_{2}+\text { etc. } \\
& +\frac{33}{2097152}\left(\frac{a}{c}\right)^{13}\left[29393 \cos ^{11} \theta_{2} \sin ^{12} \theta_{2}\right. \\
& -\frac{230945}{3} \cos ^{9} \theta_{2} \sin ^{12} \theta_{2}+72930 \cos ^{7} \theta_{2} \sin ^{12} \theta_{2} \\
& -30030 \cos ^{5} \theta_{2} \sin ^{12} \theta_{2}+5005 \cos ^{3} \theta_{2} \sin ^{12} \theta_{2} \\
& \left.-231 \cos \theta_{2} \sin ^{12} \theta_{2}+\text { etc. }\right]
\end{aligned}
$$


We must, now express all quantities in terms of $c$ and of the constants of the cylinder.

$$
\begin{aligned}
& \tan \frac{\theta}{2}=\left[\frac{1-\cos \theta}{1+\cos \theta}\right]^{\frac{1}{2}} \\
& \cos \theta_{2}=-\frac{l_{2}}{\left(c^{2}+l_{2}^{2}\right)^{\frac{1}{2}}} \text { (always negative) } \\
& \sin \theta_{2}=\frac{c}{\left(c^{2}+l_{2}^{2}\right)^{\frac{1}{2}}} \text { (always positive) } \\
& \cos \theta_{1}=\frac{l^{1}}{\left(c^{2}+l_{1}^{2}\right)^{\frac{1}{2}}} \text { (always positive) } \\
& \sin \theta_{1}=\frac{c}{\left(c^{2}+l_{1}^{2}\right)^{\frac{1}{2}}} \text { (always positive) } \\
& c^{2}+l_{1}{ }^{2}=r_{1}^{2}
\end{aligned}
$$

Substituting these values in formula (7):

$$
\begin{aligned}
& V=-2 \pi \rho a G\left(+\frac{1}{2} a \log \left[\frac{1-\cos \theta_{2}}{1+\cos \theta_{2}} \cdot \frac{1+\cos \theta_{1}}{1-\cos \theta_{1}}\right]^{1 / 2}\right. \\
& =\frac{a}{4} \log \left[\frac{\left(c^{2}+l_{2}^{2}\right)^{\frac{1}{2}}+l_{2}}{\left(c^{2}+l_{2}^{2}\right)^{\frac{1}{2}}-l_{2}} \cdot \frac{\left(c^{2}+l_{1}^{2}\right)^{\frac{1}{2}}+l_{1}}{\left(c^{2}+l_{1}^{2}\right)^{\frac{1}{2}}-l_{1}}\right] \\
& =\frac{a}{4}\left[\log \left(r_{2}+l_{2}\right)-\log \left(r_{2}-l_{2}\right)\right. \\
& \left.+\log \left(r_{1}+l_{1}\right)-\log \left(r_{1}-l_{1}\right)\right] \\
& +\frac{1}{16} a^{3}\left[\frac{l_{2}}{r_{2}^{3}}+\frac{l_{1}}{r_{1}^{3}}\right] \\
& +\frac{1}{128} a^{5}\left[-\frac{5 l_{2}^{3}}{r_{2}{ }^{7}}+\frac{3 l_{2}}{r_{2}{ }^{5}}-\frac{5 l_{1}^{3}}{r_{1}{ }^{7}}+\frac{3 l_{1}}{r_{1}^{5}}\right] \\
& +\frac{5}{2048} a^{7}\left[\frac{21 l_{2}{ }^{5}}{r_{2}{ }^{11}}-\frac{70 l_{2}^{3}}{3 r_{2}{ }^{9}}+\frac{5 l_{2}}{r_{2}{ }^{7}}\right. \\
& \left.+ \text { similar terms in } l_{1} \text { and } r_{1}\right] \\
& +\frac{7}{32768} a^{9}\left[-\frac{429 l_{2}^{7}}{r_{2}{ }^{15}}+\frac{693 l_{2}^{5}}{r_{2}{ }^{13}}-\frac{315 l_{2}{ }^{3}}{r_{2}{ }^{11}}\right. \\
& \left.+\frac{35 l_{2}}{r_{2}{ }^{9}}-\text { etc. }\right] \\
& +\frac{21}{262144} a^{11}\left[\frac{2431 l_{2}^{9}}{r_{2}{ }^{19}}-\frac{5148 l_{2}^{7}}{r_{2}{ }^{17}}+\frac{18018 l_{2}{ }^{5}}{5 r_{2}{ }^{15}}-\frac{924 l_{2}{ }^{3}}{r_{2}{ }^{13}}\right. \\
& \left.+\frac{63 l_{2}}{r_{2}{ }^{11}} \text {-etc. }\right]
\end{aligned}
$$


$V=-2 \pi \rho a G$

$$
\begin{aligned}
& {\left[\begin{array}{rl}
+\frac{33}{2097152} a^{13}[ & -\frac{29393 l_{2}{ }^{11}}{r_{2}{ }^{23}}+\frac{230945 l_{2}{ }^{8}}{3 r_{2}{ }^{21}}-\frac{72930 l_{2}{ }^{7}}{r_{2}{ }^{19}} \\
& \left.+\frac{30030 l_{2}{ }^{5}}{r_{2}{ }^{17}}-\frac{5005 l_{2}{ }^{3}}{r_{2}{ }^{15}}+\frac{231 l_{2}}{r_{2}{ }^{13}}-\text { etc. }\right]
\end{array}\right]} \\
& +\frac{429}{67108864} a^{15}\left[\frac{185725 l_{2}^{13}}{r_{2}{ }^{27}}-\frac{579462 l_{2}{ }^{11}}{r_{2}{ }^{25}}\right. \\
& +\frac{692835 l_{2}{ }^{9}}{r_{2}{ }^{23}}-\frac{2771340 l_{2}^{7}}{7 r_{2}{ }^{21}} \\
& +\frac{109395 l_{2}^{5}}{r_{2}{ }^{19}}-\frac{12870 l_{2}^{3}}{r_{2}{ }^{17}} \\
& \left.+\frac{429 l_{2}}{r_{2}^{15}}+\text { etc. }\right] \\
& +\frac{715}{2147483648} a^{17}\left[-\frac{9694845 l_{2}^{15}}{r_{2}^{31}}+\frac{35102025 l_{3}^{13}}{r_{2}{ }^{29}}\right. \\
& -\frac{50702925 l_{2}^{11}}{r_{2}^{27}}+\frac{37182145 l_{2}^{9}}{r_{2}{ }^{25}} \\
& -\frac{14549535 l_{2}^{7}}{r_{2}{ }^{23}}+\frac{2909907 l_{2}{ }^{5}}{r_{2}{ }^{21}} \\
& \left.-\frac{255255 l_{2}^{3}}{r_{2}^{19}}+\frac{6435 l_{2}{ }^{2}}{r_{2}{ }^{17}}-\text { etc. }\right] \\
& +\frac{2431}{17179869184} a^{19}\left[\frac{64822395 l_{2}^{17}}{r_{2}^{35}}-\frac{267146840 l_{2}^{15}}{r_{2}{ }^{33}}\right. \\
& +\frac{452426100 l_{2}^{13}}{r_{2}^{31}} \\
& -\frac{405623400 l_{2}^{11}}{r_{2}^{29}} \\
& +\frac{1859107250 l_{2}^{9}}{9 r_{2}{ }^{27}} \\
& -59491432 l_{2}{ }^{7} \\
& r_{2}{ }^{25} \\
& +\frac{9053044 l_{2}{ }^{5}}{r_{2}{ }^{23}}-\frac{18475600 l_{2}^{3}}{3 r_{2}{ }^{21}} \\
& \left.+\frac{12155 l_{2}}{r_{2}{ }^{19}}+\text { etc. }\right]
\end{aligned}
$$




$$
\begin{aligned}
& V=-\pi \rho a G\left(+\frac{4199}{137438953472} a^{21}\left[-\frac{883631595 l_{2}^{19}}{r_{2}^{39}}\right.\right. \\
& +\frac{4083810885 l_{2}^{17}}{r_{2}{ }^{37}} \\
& -\frac{7934261148 l_{2}{ }^{15}}{r_{2}{ }^{35}} \\
& +\frac{8415125460 l_{2}^{13}}{r_{2}{ }^{33}} \\
& -\frac{5293385370 l_{2}^{11}}{r_{2}{ }^{31}} \\
& +\frac{2007835830 l_{2}^{9}}{r_{2}{ }^{29}} \\
& -\frac{446185740 l_{2}^{7}}{r_{2}^{27}} \\
& +\frac{267711444 l_{2}^{5}}{5 r_{2}^{25}} \\
& \left.-\frac{2909907 l_{2}^{3}}{r_{2}^{23}}+\frac{46189 l_{2}}{r_{2}^{21}}-\text { etc. }\right]
\end{aligned}
$$

Differentiate with respect to $c$, noting the following relations:

and similarly:

$$
\left.\begin{array}{rl}
r_{2} & =\left(c^{2}+l_{2}^{2}\right)^{\frac{1}{2}} \\
\frac{d r_{2}}{d c} & =\frac{c}{r_{2}} \\
\frac{d r_{1}}{d c} & =\frac{c}{r_{1}}
\end{array}\right\}
$$

We obtain the following formula for the component of the force of attraction in the direction of $c$, that is, perpendicular to the axis of the cylinder:

$$
F=\frac{d V}{d c}=\pi \rho a^{2} c G\left\{\begin{array}{l}
\frac{l_{2}}{r_{2}\left(r_{2}{ }^{2}-l_{2}{ }^{2}\right)}+\frac{l_{1}}{r_{1}\left(r_{1}^{2}-l_{1}^{2}\right)}+\frac{3 a^{2}}{8}\left[\frac{l_{2}}{r_{2}{ }^{5}}+\frac{l_{1}}{r_{1}{ }^{5}}\right] \\
\quad-\frac{5 a^{4}}{64}\left[\frac{7 l_{2}{ }^{3}}{r_{2}{ }^{9}}-\frac{3 l_{2}}{r_{2}{ }^{7}}+\text { similar terms in } l_{1} \text { and } r_{1}\right] \\
+\frac{35 a^{6}}{1024}\left[\frac{33 l_{2}{ }^{5}}{r_{2}{ }^{13}}-\frac{30 l_{2}{ }^{3}}{r_{2}{ }^{11}}+\frac{5 l_{2}}{r_{2}{ }^{9}}+\text { etc. }\right] \\
-\frac{63 a^{8}}{16384}\left[\frac{715 l_{2}{ }^{7}}{r_{2}{ }^{17}}-\frac{1001 l_{2}{ }^{5}}{r_{2}{ }^{15}}+\frac{385 l_{2}{ }^{3}}{r_{2}{ }^{13}}-\frac{35 l_{2}}{r_{2}{ }^{11}}+\text { etc. }\right] \\
+\frac{231 a^{10}}{131072}\left[\frac{4199 l_{2}{ }^{9}}{r_{2}{ }^{21}}-\frac{7956 l_{2}{ }^{7}}{r_{2}{ }^{19}}+\frac{4914 l_{2}{ }^{5}}{r_{2}{ }^{17}}-\frac{1092 l_{2}{ }^{3}}{r_{2}{ }^{15}}\right.
\end{array}\right\}
$$


$+\frac{738168900 l_{2}^{13}}{r_{2}{ }^{33}}-\frac{619109400 l_{2}{ }^{11}}{r_{2}{ }^{31}}+\frac{293543250 l_{2}{ }^{9}}{r_{2}{ }^{29}}$

$-\frac{78278200 l_{2}{ }^{7}}{r_{2}{ }^{27}}+\frac{10958948 l_{2}{ }^{5}}{r_{2}{ }^{25}}-\frac{680680 l_{2}^{3}}{r_{2}{ }^{23}}$

$+\frac{12155 l_{2}}{r_{2}{ }^{21}}+$ etc. $]$

$-\frac{88179 a^{20}}{68719476736}\left[\frac{1641030105 l_{2}^{19}}{r_{2}^{41}}\right.$

$-\frac{7195285845 l_{2}{ }^{17}}{r_{2}{ }^{39}}+\frac{13223768580 l_{2}{ }^{15}}{r_{2}{ }^{37}}$

$-\frac{13223768580 l_{2}^{13}}{r_{2}^{35}}+\frac{7814045070 l_{2}^{11}}{r_{2}^{33}}$

$-\frac{2772725670 l_{2}{ }^{9}}{r_{2}{ }^{31}}+\frac{573667380 l_{2}{ }^{7}}{r_{2}{ }^{29}}-\frac{63740820 l_{2}{ }^{8}}{r_{2}{ }^{27}}$

$+\frac{3187041 l_{2}^{3}}{r_{2}{ }^{25}}-\frac{46189 l_{2}}{r_{2}^{23}}+$ etc. $]$

As an illustration of the rapidity with which the series (11) converges, take the following simple case of the attraction of a cylinder upon a point at the middle of its lateral surface:

$$
\begin{gathered}
a=c=l_{1}=l_{2}=10 \\
r_{2}=r_{1}=10 \sqrt{2}
\end{gathered}
$$


Omitting the factor $\pi \rho c G$, the successive terms and their sums are shown in Table 5.

TABLE 5.-Convergency of series

\begin{tabular}{|r|r|r|}
\hline Term & Value of term & Sum of series \\
\cline { 1 - 1 } 1 & +1.4142136 & 1.4142136 \\
2 & +.1325825 & 1.5467961 \\
3 & -.0069053 & 1.5398908 \\
4 & -.0052869 & 1.5346039 \\
5 & +.0005735 & 1.5351774 \\
6 & +.0005233 & 1.5357007 \\
7 & -.0000708 & 1.5356299 \\
8 & -.0000698 & 1.5355601 \\
9 & +.0000105 & 1.5355706 \\
10 & +.0000108 & 1.5355814 \\
11 & -.0000017 & 1.5355797 \\
\hline
\end{tabular}

For points farther distant from the axis of the cylinder the convergence is more rapid.

Equation (11) may seem formidable, but it is comparatively simple in practical application. Inspection of the formula shows that, as often happens in series, the first term is apparently anomalous, but that beginning with the second term certain laws are discoverable.

Disregarding for the moment the numerical factors, it will be seen that within the parentheses we have a series of literal terms containing $l_{2}$ and $r_{2}$ followed by an exactly similar series containing $l_{1}$ and $r_{1}$. Moreover, as we pass down the terms of the whole equation, we find (still disregarding the numerical factors) that there are certain moduli or multiplying factors by which any term can be converted into the next following. An example will make this clear. Take the second, third, and fourth terms of (11), bringing in the factor $a^{2}$ from the general factor $\pi \rho a^{2} c G$ :

$$
\left.\begin{array}{ll}
+\frac{a^{4} l_{2}}{r_{2}{ }^{5}} ; & +\frac{a^{4} l_{1}}{r_{8_{1}^{5}}} \\
-\frac{a^{6} l_{2}{ }^{3}}{r_{2}{ }^{9}}+\frac{a^{6} l_{2}}{r_{2}{ }^{7}} & -\frac{a^{6} l_{1}^{3}}{r_{1}{ }^{9}}+\frac{a^{6} l_{1}}{r_{1}{ }^{7}} \\
+\frac{a^{8} l_{2}^{5}}{r_{2}{ }^{13}}-\frac{a^{8} l_{2}^{3}}{r_{2}^{11}}+\frac{a^{8} l_{2}}{r_{2}{ }^{8}} ; & +\frac{a^{8} l_{1}^{5}}{r_{1}^{13}}-\frac{a^{8} l_{1}{ }^{3}}{r_{1}^{11}}+\frac{a^{8} l_{1}}{r_{1}{ }^{9}}
\end{array}\right\}
$$

It will be noticed first that the term heading every column is positive, and that the signs alternate as we go down the column. Moreover, the terms in each column have a common ratio, $\frac{a^{2} l_{2}^{2}}{r_{2}{ }^{4}}$ or $\frac{a^{2} l_{1}^{2}}{r_{1}{ }^{4}}$ as the case may be. This ratio may be called the vertical modulus.

There is also a common ratio running diagonally downward and to the right, $\frac{a^{2}}{r_{2}{ }^{2}}$ or $\frac{a^{2}}{r_{1}{ }^{2}}$, which we may call the diagonal modulus. And there will be consequently a similar horizontal modulus, $\frac{r_{2}^{2}}{\bar{l}_{2}^{2}}$ or $r_{1}{ }^{2}$. Hence, given the second literal term, each succecding literal 
term may be constructed by means of the vertical and diagonal moduli and checked by the horizontal modulus; and since cuch modulus is concerned only with $a, r$, and $l$ (constants of the cylinder and of the position of the balls), these moduli may be calculated once for all the beginning of the computation.

In addition to these moduli we have the numerical coefficients, unity in the first term, three-eighths in the second, and so on. In the third and later terms we have more than one coefficioni. 'Table 5 gives these coetficients for the first seven terms to a sufficient number of decimal places to secure an accuracy of 1 part in $100,(0)(0)$ in the value of the attraction when $a=10 \mathrm{~cm}, l_{1}+l_{2}=28 \mathrm{~cm}$, and $c=13$ $\mathrm{cm}$, which correspond closely to the actual working conditions.

TABLE 6.-Working coefficients

\begin{tabular}{|c|c|c|c|c|c|c|}
\hline Term & \multicolumn{6}{|c|}{ Coefflicients } \\
\hline $\begin{array}{l}1 \\
2\end{array}$ & & & & & & \\
\hline $\begin{array}{l}2 \\
3 \\
4\end{array}$ & $\begin{array}{r}+0.37500 \\
-.546875 \\
\end{array}$ & $\begin{array}{l}+0.234375 \\
-1\end{array}$ & & & & \\
\hline 5 & -2.7493 & $\begin{array}{l}-1.0491 \\
+3.841\end{array}$ & -1.4804 & +0.13458 & & \\
\hline $\begin{array}{l}6 \\
7\end{array}$ & $\begin{array}{c}+7.400 \\
-21.28\end{array}$ & $\begin{array}{l}-14.022 \\
+50.88\end{array}$ & $\begin{array}{c}+8.6604 \\
-43.609\end{array}$ & $\begin{array}{l}-1.9245 \\
+16.066\end{array}$ & $\begin{array}{l}{ }_{-2.363}^{0.1110} \\
\end{array}$ & +0.0945 \\
\hline
\end{tabular}

The steps in the computation of the force of attraction of a finite cylinder at any external point may now be summarized as follows:

1. From $a, r_{1}, r_{2}, l_{1}, l_{2}$, and $c$, calculate the rertical, diagonal, and horizontal moduli.

2. Calculate the first literal term of (11).

3. Calculate the second literal term of (11).

4. By means of the moduli calculate the succeeding literal terms (exclusive of the numerical factors).

5. Complete the calculation by applying to the literal terms the proper numerical factors from Table 6.

\section{FORMULA FOR TIME OF SWING}

We have to deal with a torsion pendulum swinging in a nonuniform but symmetrical gravitational field, the axis of symmetry, in the near position of the masses, coinciding with the axis of the moving system, and in the far position being at right angles to this axis. Were the field uniform and parallel the formula for the time of swing would be

$$
T=2 \pi \sqrt{\frac{I}{\tau+\Sigma(F R)}}
$$

in which $I$ is the moment of inertia of the moving system and $\tau+\Sigma(F R)$ the restoring moment per unit angular displacement. In this latter expression $\tau$ is the part contributed by the modulus of torsion of the filament and $\Sigma(F R)$ the part arising from the gravitational field.

It might be thought that the effect of the nonuniformity of the gravitational field would disappear in the reduction of the time of 
swing to zero amplitude. As a matter of fact, such a procedure gives a value of the constant of gravitation about 60 per cent too great. As will shortly be shown we must substitute for $R$ in (13) the quan-

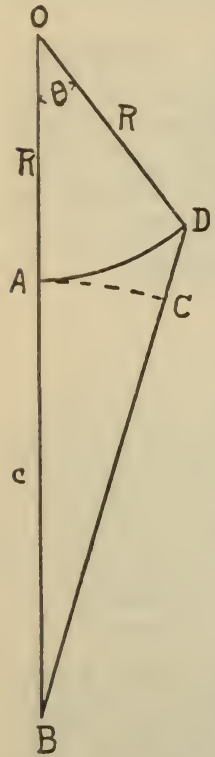

FigURe 7.-Close ball plan, near position tity $R(1+R / c)$ in the near position, where $R / c$ is approximately $10 / 13$, and in the far position a much more complicated factor is necessary. We, therefore, have instead of $\Sigma$ $(F R)$ in (13) a quantity $\Sigma(F K)$, where $K$ is a more or less complicated function of $R$, and the working formula for the time of swing becomes:

$$
T=2 \pi \sqrt{\frac{I}{\tau+\Sigma(F K)}}
$$

This equation, together with the factors $K$ for the near and the far position, may be derived as follows. We assiume the cylinder axes parallel and vertical. Figures 7 and 8 show sections perpendicular to the axis of the cylinder. In these figures let

$B=$ axis of cylinder.

$O=$ center of rotation of ball.

$A=$ neutral position of ball when $\theta=0$.

$D=$ displaced position of ball.

$c=$ distance between centers of ball and cylinder in neutral position of ball $=A B$.

For the near position and for the ball close to the cylinder (fig. 7):

$$
\begin{aligned}
B D & =\left[(O A+A B)^{2}+\bar{O} \bar{D}^{2}\right. \\
& -2(O A+A B) O D \cos \theta]^{\frac{1}{2}} \\
& =\left[(R+c)^{2}+R^{2}-2(R+c) R \cos \theta\right]^{\frac{1}{2}} \\
& =c\left[1+\frac{2 R}{c}\left(1+\frac{R}{c}\right)(1-\cos \theta)\right]^{1}
\end{aligned}
$$

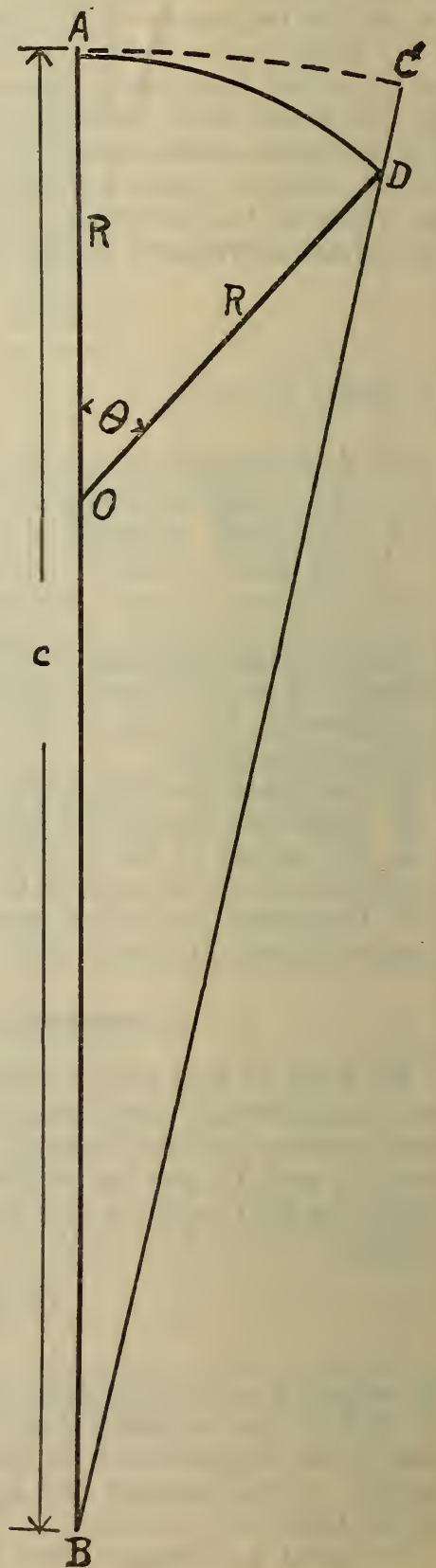

FIGURE 8.-Distant ball plan, near position 
Expanding by the binomial theorem:

$$
\begin{aligned}
B D & =c\left[1+\frac{R}{c}\left(1+\frac{R}{c}\right)(1-\cos \theta)\right. \\
& \left.-\frac{1}{2} \frac{R^{2}}{c^{2}}\left(1+\frac{R}{c}\right)^{2}(1-\cos \theta)^{2}+\cdots \cdots\right]
\end{aligned}
$$

$D C=B D-B C=B D-c$

$$
=c\left[\frac{R}{c}\left(1+\frac{R}{c}\right)(1-\cos \theta)-\frac{1}{2} \frac{R^{2}}{c^{2}}\left(1+\frac{R}{c}\right)^{2}(1-\cos \theta)^{2}+\cdots\right]
$$

When $\theta$ is small

$$
1-\cos \theta=\frac{\theta^{2}}{2} ; \quad(1-\cos \theta)^{2}=\frac{\theta^{4}}{4}
$$

Neglecting small terms involving the fourth and higher powers of $\theta$ :

$$
L=\mathrm{Lift}=D C=c\left[\frac{1}{2} \frac{R}{c}\left(1+\frac{R}{c}\right) \theta^{2}\right]
$$

Let $F_{0}$ denote the force of attraction upon the ball at $A$, and $F_{\theta}$ that upon the ball when at $D$. The force $F$ will be a function of the distance $B D$ between centers of cylinder and ball, though not exactly proportional to $1 / B D^{2}$, since the cylinder is not barycentric. This variable distance $B D$ will differ slightly from the small quantity $D C=L$ by the constant $c$; hence $d(B D)=d L$.

Expand $F_{\theta}$ as a function of $B D$ by Taylor's theorem:

in which

$$
\begin{aligned}
F_{\theta} & =F_{0}+L \frac{d F}{d L}+\frac{L^{2}}{2} \frac{d^{2} F}{d L^{2}}+\ldots \ldots \\
& =F_{0}\left[1+V L+V \cdot L^{2}+\ldots \ldots\right]
\end{aligned}
$$

$$
V=\frac{1}{F_{0}} \frac{d F}{d L} \text { and } V_{1}=\frac{1}{2 F_{0}} \frac{d^{2} F}{d L^{2}}
$$

Since $F$ decreases with increase of $B D, \frac{d F}{d L}$ is negative; and since the rate of decrease of $F$ diminishes with increase of $B D$ (the $F$ curve being concave upward) $\frac{d^{2} F}{d L^{2}}$ is positive. The usual values for these quantities were about -0.07 for $V$ and +0.0015 for $V_{1}$. We shall find it convenient to write:

$$
\text { Force at } D=F_{\theta}=F_{0}\left[1-V L+V_{1} L^{2}+\ldots\right]
$$

considering both $V$ and $V_{1}$ as positive. For the case of the close ball which we are considering $L$ is also positive.

As the ball passes from $A$ to $D$ its differential increase in potential energy will be:

$$
F_{\theta} d L=F_{0}\left[d L-V L d L+V_{1} L^{2} d L+\ldots\right]
$$


For the small angle $\theta$ and the small change in $B D$ contemplated $V$ and $V_{1}$ will vary but little. Integrating the last equation we obtain:

$$
\text { Potential energy }=F_{0}\left[L-\frac{1}{2} V L^{2}+\frac{1}{3} V_{1} L^{3}\right]
$$

Applying (15):

$$
\begin{aligned}
\text { Potential energy } & =F_{0}\left[\frac{c}{2}\left\{\frac{R}{c}\left(1+\frac{R}{c}\right)\right\} \theta^{2}\right]+\ldots \\
& =\frac{1}{2} F_{0} R\left(1+\frac{R}{c}\right) \theta^{2}
\end{aligned}
$$

For the distant ball (fig. 8) proceeding similarly:

$$
B D=c\left[1-\frac{2 R}{c}\left(1-\frac{R}{c}\right)(1-\cos \theta)\right]^{\frac{1}{2}}
$$

Expanding and neglecting small terms:

$$
\begin{aligned}
B D & =c\left[1-\frac{R}{c}\left(1-\frac{R}{c}\right)(1-\cos \theta)\right] . \\
D C & =B D-c \\
& =c\left[-\frac{R}{c}\left(1-\frac{R}{c}\right)(1-\cos \theta)\right] \\
L & =\operatorname{Lift}=D C=-c\left[\frac{1}{2} \frac{R}{c}\left(1-\frac{R}{c}\right) \theta^{2}\right]
\end{aligned}
$$

Force at $D=F_{\theta}=F_{0}\left[1-V L d L+V_{1} L^{2} d L\right]$

in which $V$ and $V_{1}$ are considered positive and, for the distant ball, $L$ is negative.

$d$ (potential energy) $=F_{\theta} d L=F_{0}\left[d L-V L d L+V_{1} L^{2} d L\right]$

$$
\begin{aligned}
\text { Potential energy } & =F_{0}\left[L-\frac{1}{2} V L^{2}+\frac{1}{3} V_{1} L^{3}\right] \\
& =F_{0}\left[-\frac{1}{2} c \frac{R}{c}\left(1-\frac{R}{c}\right) \theta^{2}\right] \\
& =-\frac{1}{2} F_{0} R\left(1-\frac{R}{c}\right) \theta^{2}
\end{aligned}
$$

Since (16) and (17) are identical except for sign of $R$, we may regard $R$ as positive for the close ball and negative for the distant ball, and 
with this understanding write for the potential energy of a cylinderball system in the near position:

$$
+\frac{1}{2} F_{0} R\left(1+\frac{R}{c}\right) \theta^{2}
$$

A typical case of the values which (18) may give may be cited from the platinum ball set. Denoting the two cylinders by $E$ and $W$, the ball close to cylinder $E$ by $A$ and the ball close to cylinder $\|^{\circ}$ by $B$, we have:

$$
\begin{aligned}
& E A=+228179 G \theta^{2} \\
& E B=-19417 G \theta^{2} \\
& W A=-19562 G \theta^{2} \\
& W B=+233141 G \theta^{2}
\end{aligned}
$$

The far position is illustrated in Figures 9 and 10, in which the letters have the same meaning as in Figures 7 and 8 . By the close ball

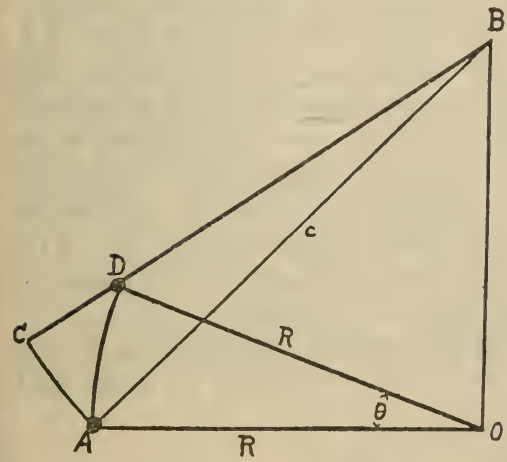

Figure 9.-Close ball plan, far position

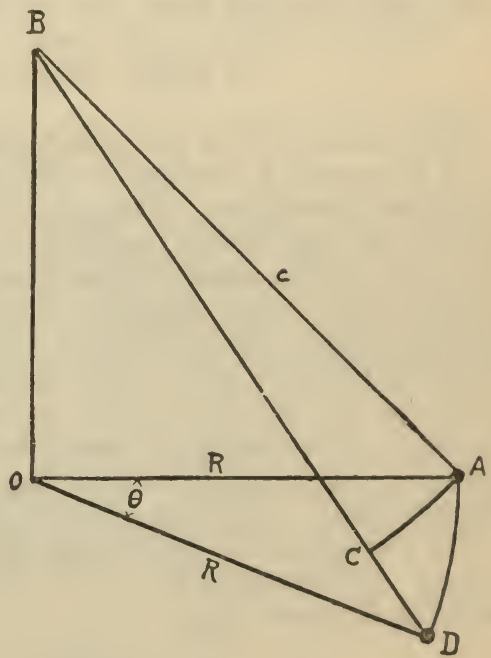

Figdre 10.-Distant ball plan, far position

in this case is meant that ball which is swinging from $A$ to $D$ toward the cylinder at $B$. Proceeding as in the near position, but keeping terms including $\theta^{3}$, we find for the potential energy of the system including both cylinders and both balls:

$$
\begin{aligned}
\text { Potential energy }= & -\Sigma\left[F_{0} R\left(1-\frac{R^{2}}{c^{2}}\right)^{\frac{1}{2}}\right] \theta \\
& -\frac{1}{2} \Sigma\left[F_{0}\left(\frac{1+V^{2} c}{c}\right) R^{2}\left(1-\frac{R^{2}}{c^{2}}\right)\right] \theta^{2} \\
& \left.-\frac{1}{2} \Sigma\left[F_{0}\left(\frac{1+V c+\frac{2}{c^{2}} V^{2} r^{2}}{c^{2}}\right) R\left(1-\frac{R^{2}}{c^{2}}\right)^{2}\right] \beta\right]
\end{aligned}
$$


Each sum consists of four terms, of signs determined as follows. $\theta$ is always assumed positive, the two cylinders are designated as $N$ and $S$ (having now been turned $90^{\circ}$ from the near position), the ball close to the $N$ cylinder is called $A$ and the ball close to the $S$ cylinder $B$, the term close being used in the sense defined above. The signs of the different terms within the summation are given in Table 7.

TABLE 7.-Signs of terms in formula (19)

\begin{tabular}{|c|c|c|c|}
\hline Term & $\begin{array}{c}\text { Coeffi- } \\
\text { cient of } \theta\end{array}$ & $\begin{array}{c}\text { Coeffi- } \\
\text { cient of } \theta_{2}\end{array}$ & $\begin{array}{c}\text { Coeffi- } \\
\text { cient of } \theta^{3}\end{array}$ \\
\hline$N A$ & \pm & \pm & \pm \\
$N B$ & \pm & \pm & \pm \\
$S A B$ & \pm & \pm \\
\hline
\end{tabular}

In formula (19) the most important term is that in $\theta^{2}$. A typical case may be cited from the platinum ball set.

$$
\text { 6. } 78 G \theta-87199.42 G \theta^{2}+64.63 G \theta^{3}
$$

The term in $\theta^{3}$ is negligible, jointly because of the small value of $\theta$ and the small numerical coefficient, but the presence of the term in $\theta$ requires attention. Formula (20) represents the potential energy of the moving system, and this should be independent of the sign of $\theta$. It will be remembered that the moving system was centered in azimuth in the near position, and that this setting was of necessity approximate. In passing to the far position the cylinders were rotated through $90^{\circ}$. Had the original azimuth centering been accurate the far position would be one of equilibrium, and the potential energy when displaced would be independent of the sign of $\theta$. The presence of a term in $\theta$ shows that the far position is not exactly one of equilibrium. We may determine this error as follows.

In a position of equilibrium the potential energy should be a maximum. Differentiating (20) with respect to $\theta$, equating to zero and solving for $\theta$ we find:

$$
\theta=0.00003888 \text { radian }=8.02^{\prime \prime}
$$

This correction is so small that we might feel justified in dropping the term in $\theta$ at once; but to confirm this impression we substitute in (20) for $\theta$ the corrected value $\theta+0.00003888$, and we find that the coefficient of $\theta$ now becomes zero as it should, while the coefficient of $\theta^{2}$ is 87199.40 , very near the value in (20). In consequence we have to consider in the far position as well as in the near only the term in $\theta^{2}$.

The formula for the time of swing (14) may now be derived. For any displacement $\theta$ from the equilibrium point:

Kinetic energy of pendulum + potential energy due to filament + potential energy of attraction = constant.

Let $I=$ moment of inertia of pendulum.

$\tau=$ modulus of torsion of filament (moment required to give the beam an angular displacement of one radian) 
and (21) becomes:

$$
\frac{I}{2}\left(\frac{d \theta}{d t}\right)^{2}+\frac{1}{2} \tau \theta^{2}+\frac{1}{2} \leq(F K) \theta^{2}=\text { constant }
$$

By reference to (16) and (17) we see that the values of $K$ for the close and the distant balls in the near position are respectively

$$
R\left(1+\frac{R}{c}\right) \text { and }-R\left(1-\frac{R}{c}\right)
$$
In the far position we find the values of $K$ by reference to (19) and
Table 7 .

Differentiating and reducing:

$$
\frac{d^{2} \theta}{d t^{2}}+\frac{\tau+\Sigma(F K)}{I} \theta=0
$$

an equation of the usual form for harmonic motion with a period:

$$
T=2 \pi \sqrt{\frac{I}{\tau+\Sigma(F K)}}
$$

In the foregoing derivation it has been assumed that the cylinder axes are vertical. A more general calculation of a similar nature for inclined axes shows that if the inclination of either axis is less than $15^{\prime}$ the error introduced by considering the axis vertical is less than 1 part in 100,000. The actual inclination in any experiment was much less than this, hence the formulas for vertical axes have been used throughout the computations.

Formula (14) applied to the near and far positions gives us two equations for the two unknown quantities $\tau$ and $G$, which latter quantity appears in F. (See formula (11).) Eliminating $\tau$ from these two equations we obtain a formula for $G$.

\section{METHOD OF OBSERVING}

\section{CENTERING ADJUSTMENTS}

In the foregoing discussion it is assumed that the center of rotation of the moving system is at the middle point of a straight line joining the axes of the cylinders. The fulfilling of this condition requires three centering adjustments, azimuth, longitudinal and transverse, as indicated in Figure 11. These adjustments are best made in the near position of the cylinders.

The azimuth adjustment was made by observing the time of swing at various angular settings of the cylinders. If the moving system is centered transversely, the curve of time of swing against azmuth will have a single minimum. If the transverse adjustment is sufficiently off two minima will be present.

A typical azimuth centering curve is shown in Figure 12. Such curves were usually characterized by a rather broad and flat minimum; a well-marked double minimum was rare. The minimum 
point is best determined by means of pairs of points on the steep slopes, and could usually be fixed to one or two tenths of a degree.
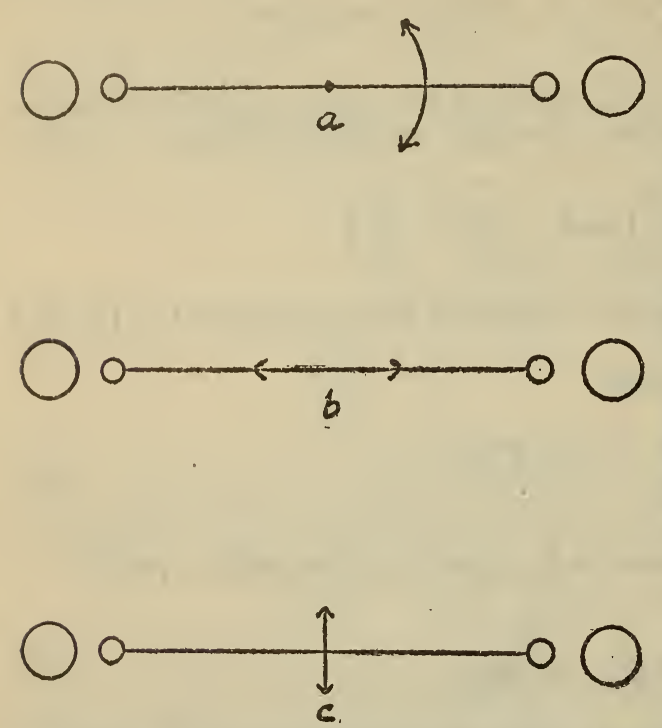

Frgure 11.-Centering adjustments $a$, Azimuth; $b$, longitudinal; $c$; transverse.

So flat is the minimum that such an accuracy is quite sufficient.

Having made a first adjustment for azimuth, the next step was to adjust transversely. This adjustment might have been made by direct observation of the filament, but it was more convenient to work by time of swing. The desired point is marked here, as in azimuth, by a minimum or, in case the azimuth centering is out, by a double minimum.

The longitudinal setting was made by observing the filament through windows in the chimney. The curve for time of swing in this case would be characterized by a maximum. Such a curve was actually obtained as a check, but the maximum was so very flat that it was impossible to locate the desired point in this way within several millimeters.

After a first adjustment of all three kinds, the azimuth and transverse settings were repeated, sometimes twice. The longitudinal adjustment, having a very flat maximum, required little precision.

Theoretically, no further adjustmentwould be required in the far position. The new azimuth could be set by the scale and vernier to $0.1^{\circ}$, a precision equal to that obtainable by centering. As to the other adjustments, a little consideration will show that the maximum or minimum must be much flatter than in the near position, and that

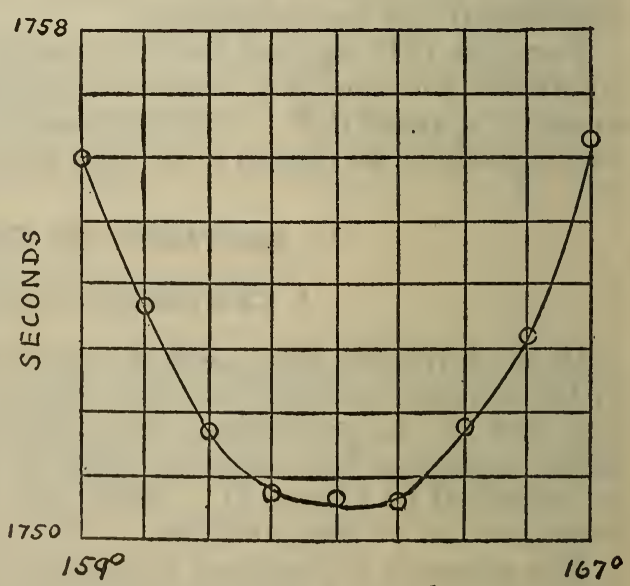

FigURE 12.-A zimuth centering curve these adjustments consequently require less precision in the far than in the near position.

In practice the transverse and longitudinal adjustments were made by moving the sliding dovetailed blocks which held up the beam $B$. 
(Fig. 3.) Such shifts of course necessitated further shifts for correction of the transverse and longitudinal adjustments after turning the cylinders into the far position. To provide for this the dovetailed blocks carried millimeter scales by means of which it was possiblo to recover the center promptly.

\section{LENGTH MEASUREMENTS}

(a) HORIZONTAL

The horizontal measurements necessary were: (1) Distance botween centers of balls, and (2) distance between axes of cylinders.

From these may be calculated the distances from centers of balls to axes of the two cylinders, the quantity $c$ entering into the formula for the force of attraction. In practice the second of these measurements was replaced by the distance between the nearest elements of the surfaces of the cylinders. This, in conjunction with the radii of the cylinders, gave the desired quantity.

The distance between centers of balls had to be measured with the lid of the container lifted. This measurement was therefore made twice, before the container was closed and again, some months later, at the conclusion of the campaign, when the container was reopened. In measuring this distance the microscopes of the optical compass were sighted on the tungsten filaments by which the balls were suspended, and at a point as far above the balls as possible (about $3 \mathrm{~cm}$ ). Settings were made on both sides of the filament (diameter $0.025 \mathrm{~mm}$ ) and the mean value assumed to represent the point of suspension, vertically above the center of gravity of the ball.

The distance between cylinders was the most troublesome and time consuming of all the length measurements. The first thing was to set the cylinders with their axes approximately vertical. To prevent swaying of the cylinders a small block of aluminum weighing about $1 \mathrm{~g}$ was placed between container and cylinders at the bottom of the latter and fastened in place by a little wax. Similar blocks were attached to the container at points $90^{\circ}$ from the first blocks, to be used in the far position of the cylinders. In changing the position of the cylinders the blocks were left in place, and consequently played no part in the computation.

The cylinders thus fixed at the bottom were adjusted by the turnbuckles guided by a small plumb bob with a fine thread. Calculation showed that a variation of $15^{\prime}$ from the vertical was allowable with a precision of 1 part in 100,000, and the actual error was always much less than this.

Direct sighting of the microscopes tangentially upon the surface of the cylinders being impracticable, the following device was found to give satisfactory results.

In a piece of straight brass rod about $1.5 \mathrm{~mm}$ in diameter there was inserted the point of a fine steel needle. (Fig. 13.) This rod was let down into the space between the container and one of the cylinders, and supported by a screw passing through a heavy pieco of brass which, in its turn, was supported by a bluck of wood fastened temporarily with wax to the lid of the container. A similur arrangement was provided for the other cylinder: Bruss rods of two different lengths were provided in order that the distance between the cylindery might be measured near the upper and lower ends. 
In order to record and reproduce the position of the brass rod each wooden block was provided on the top with a short piece of paper scale across which lay the piece of metal supporting the brass rod.

It was not difficult to adjust the needle perpendicular to the line of sight with sufficient accuracy. The magnifying power of the microscope was such that any serious departure in this respect showed itself at once by an impairment of focus at the ends of the needle, and the apparent change of length being a cosine function the adjustment was one which did not require the highest precision.

The needle was first hung a little distance from the cylinder so that its point was visible, and a sighting mark sought on the needle some little distance back of the point. If there was no slight irregularity

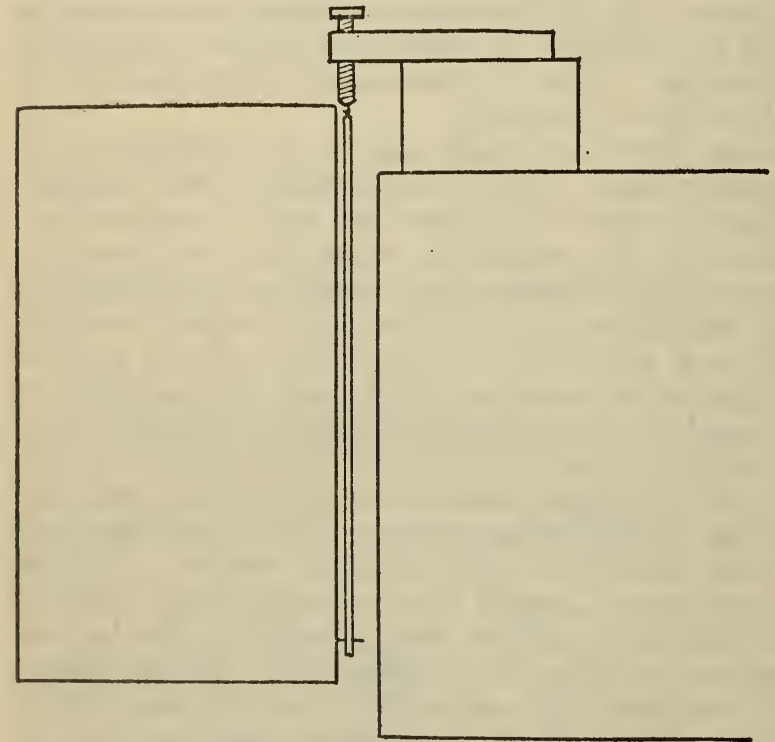

FIGURE 13.-Sighting mark for determining distance available on the needle one was made by a slight touch of a file. The distance from this sighting mark to the point of the needle (in terms of turns of the micrometer) was recorded for each needle immediately before use.

The needle points were now placed in contact with the cylinders at a determined level and settings made upon the sighting marks.

The distance required is evidently the minimum distance that can be found by setting the two needles in various positions. The following systematic procedure was adopted.

With the needle point on the left in one fixed position the one on the right was moved step by step along the paper scale until a minimum reading was obtained. It was not difficult to obtain a symmetrical set of readings in this way. An example is given in Table 8.

TABLE 8.-Determination of minimum distance

\begin{tabular}{|c|c|c|}
\hline Left needle & $\begin{array}{c}\text { Right } \\
\text { needle }\end{array}$ & Distance \\
& & $c m$ \\
18.3 & 15.5 & 27.8527 \\
18.3 & 15.6 & 27.8484 \\
18.3 & 15.7 & 27.8476 \\
18.3 & 15.8 & 27.8485 \\
18.3 & 15.9 & 27.8530 \\
\hline
\end{tabular}


Such a series of measurements could usually be repeated with an accuracy better than $0.001 \mathrm{~cm}$.

Having located a minimum on one side in this way the needle was set at this place and a minimum sought with the other needle. After having found this, a second setting for minimum was agrin made on each side, resulting usually in a very small correction.

As a check of this method the diameter of one of the cylinders was measured at several places. The mean agreed with that obinined by the weights and measures division of the bureau to $5 \mu$.

(b) VERTICAL

The vertical measurements necessary were those involved in the relative positions of centers of balls and tops of cylinders, the inclination of the aluminum rod to the horizontal and the altitude of the triangular truss. (Fig. 4.) These measurements were made with a kathetometer to a precision of about $0.1 \mathrm{~mm}$. For the relative level of balls and cylinder tops measurements were taken through the windows in the container at the conclusion of each determination of time of swing. For the other two measurements, observations were made with the lid of the container lifted.

\section{TIME MEASUREMENTS}

In making measurements of the time of swing the general plan used by Braun was followed.

On the day before a series of time observations was intended the resting point of the moving system was adjusted so as to lio as near as possible to the $12.5 \mathrm{~cm}$ mark. On the observing day the initial resting point was noted and the pendulum started swinging by means of the mercury bottles. Since the swings always passed off the scale, the successive amplitudes were watched and controlled by observing the speed with which the centimeter containing the resting point passed the cross wire of the telescope. The amplitudes of any two successive swings are proportional to the central speeds preceding (or following) the peak of the swing.

The multiplying factor in this case was determined by observations made on swings small enough to allow direct measurements of the amplitude. The results are given in Table 9. In this table the central speed is the speed at which the image of the scale passed the cross wire of the observing telescope, and the amplitude is that of the deflection of the image of the scale.

TABLE 9.-Relation between amplitude and central spced

\begin{tabular}{|c|c|c|c|c|}
\hline $\begin{array}{l}\text { Scale reading } \\
\text { in centimeters }\end{array}$ & $\begin{array}{c}\text { Resting } \\
\text { point }\end{array}$ & $\begin{array}{l}\text { Central } \\
\text { speed }\end{array}$ & Amplitude & $\frac{\text { Amplitude }}{\text { Speed }}$ \\
\hline 4.68 & \multirow[b]{2}{*}{11.00} & $\mathrm{~cm} / \mathrm{min}$. & & \\
\hline 16.93 & & 102 & 5.93 & 5.8 \\
\hline 5.44 & \multirow{2}{*}{$\begin{array}{l}11.04 \\
11.01\end{array}$} & .97 & 5. 60 & 5.8 \\
\hline 16.22 & & .92 & 5. 21 & 5.7 \\
\hline \multirow[t]{2}{*}{6.15} & \multirow[t]{2}{*}{11.01} & \multirow[t]{2}{*}{.86} & \multirow[t]{2}{*}{ 4. 80} & 5.7 \\
\hline & & & & Mean._ 5.7 \\
\hline
\end{tabular}


It was found possible to bring up the central speed to about 9 $\mathrm{cm} / \mathrm{min}$. in about two hours by repeated changing of the positions of the mercury bottles. This speed corresponds to an amplitude of about $51 \mathrm{~cm}$. Since the radius of the reflected ray was about 350 $\mathrm{cm}$ this amplitude corresponded to an angular deflection of about $4^{\circ}$. The actual amplitudes employed in the measurements ranged from something near this figure at starting to perhaps $1 \frac{1}{\circ}$ at the end of a day's observing.

Having obtained the desired initial amplitude the mercury bottles were removed, the temperature and pressure within the container noted, and the apparatus room darkened. The observer then took his seat at the telescope in the observing room and observed 24 successive transits, recording on the chronograph the time of transit of each millimeter mark from 11.0 to $14.0 \mathrm{~cm}$. This required six hours in the near position and seven in the far position, during which time the observer remained in the same place.

By the time the observations were finished the central speed had diminished to about one-third of its initial value. If the final speed was slow, it was difficult to time the transits accurately; if, on the other hand, the initial speed was too high a large reduction to zero amplitude had to be made in the subsequent computation. Such a reduction involves an extrapolation which it is desirable to keep as small as possible. These two modes of procedure, high and low initial speeds, were both used, the initial speeds with the glass ball set being about 10 per cent higher than those with the platinum balls.

The form of the amplitude-time curve for the pendulum was not strictly that of the form $y=A e^{=b t}$ sin $c t$, and because the pendulum moved in a nonuniform gravitational field and because of the damping factor, was not exactly isochronous. This difference amounted at most to about 1 part in 3,000. Strictly speaking, the method of reduction employed by Braun and in the present work is not applicable to a nonisochronous curve of this description, but the departure from isochronism was so small and the damping factor so nearly unity that it was found that results sufficiently accurate could be obtained in the manner about to be described.

The first step was the determination of the damping factor. By inspection of the chronograph records the middle point of a swing could be approximately located as the point where the intervals between consecutive transits were equal. At this point of the scale the ratio of the central speeds for any two successive swings in the same direction gave the damping factor. This proved to be about 0.9 , and was very nearly constant throughout any given day's run. The absolute value of this factor varied from day to day with variations of pressure in the container, being in fact a far more sensitive indicator of change of pressure than the manometer. The constancy of the damping factor for any given run showed that the successive amplitudes of the curve followed a geometrical progression.

The second step was a more accurate location of the middle point of the swings. This can be done with considerable precision by the procedure indicated in 'Table 10 . In this table the column headed II contrins the difference of the numbers in columns 2.5 and 1.5 . Where the numbers in Column II change sign is the middle point, and from this column four values of the middle point are obtainable, interpolating between scale readings 12.2 and $12.3 ; 12.1$ and 12.4; 
12.0 and $12.5 ; 11.9$ and 12.6 . These four values are set down belnw Column II, and their mean, 12.265 , is taken us representing the middle point during swings 1 and 2. Similarly, swings 2 and 3 yield the almost identical value 12.269 .

Any large departure from isochronism would bring about a considerable difference in the two middle points just calculated. Middle points later in the series, where the departure from isochronism is less, would show less and less difference.

It was customary to calculate the middle point only at the beginning and end of a run, as indicated in Table 10. It will be seen that in this case there has been a drift of the middle point amounting to 0.13 $\mathrm{cm}$ during the day's observations. Where the drift was as small as this it was customary to interpolate linearly between the initial and final values to obtain the middle points throughout the run; but if there was any suspicion of irregularity or sudden change this calculation was repeated all through the series.

TABLE 10.-Calculation of middle point

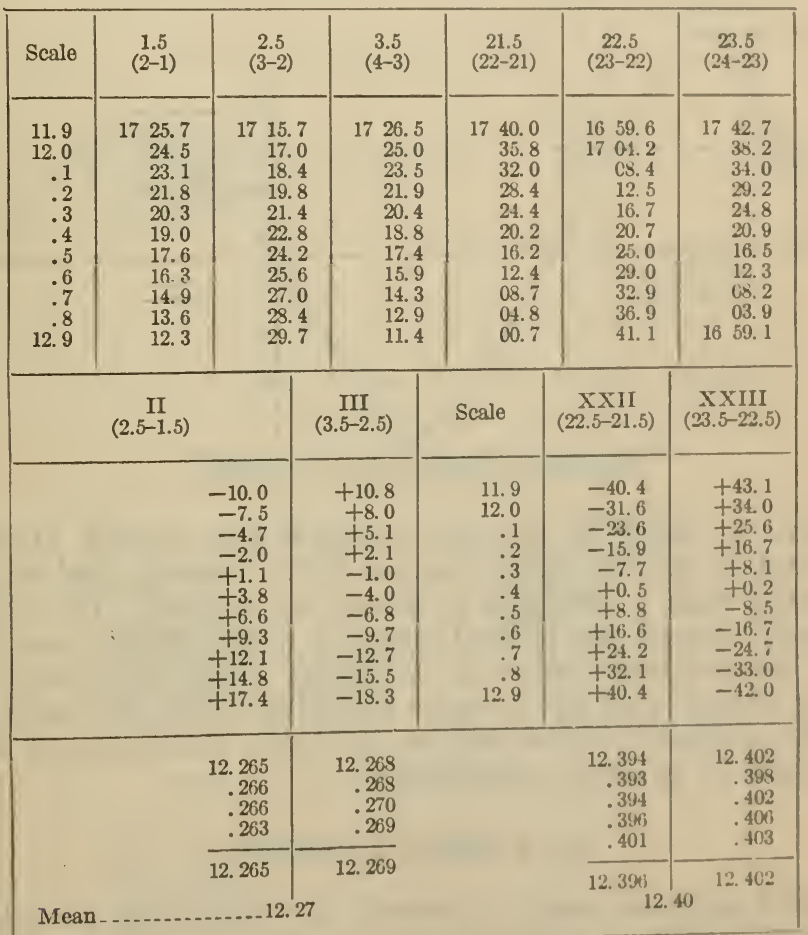

The third step was the application of sinusoidal corrections. In a curve of the form $y=A e^{-b t} \sin c t$, to which the actual curve closely approximates (fig. 14), the period can be found by the interval between intersections with the axis $O t$, for which the amplitude $y=0$, but not from intersections with any other line parallel to Ot, such as $a_{1} a_{2}$. The point $a_{2}$ must be brought down to its proportionate level $a_{3}$ by the application of the damping factor, after which the period may be 
found by the difference between the values of $t$ for $a_{1}$ and $a_{3}$. In this way values of the period may be obtained for every recorded transit of a mark on the scale, whether at the middle point or not, and a mean value of considerable precision obtained.

From 24 transits we may thus obtain 22 values of the period, corresponding to steadily decreasing amplitudes of the curve. Of these periods, 11 are obtained from transits occuring on up slopes of the curve and 11 on down slopes. Theoretically, in a sinusoid these two slopes differ slightly in form, and, consequently, the periods derived from the two slopes will differ slightly. For this reason the 22 periods were reduced to 11 by taking means of consecutive pairs of up and down values of the period.

After correction of these 11 values for damping they were reduced to zero amplitude. For this purpose an equation was used which ininvolved the second power of the amplitude. From the 11 values of the period normal equations were set up, and solved for the reduced time of swing.

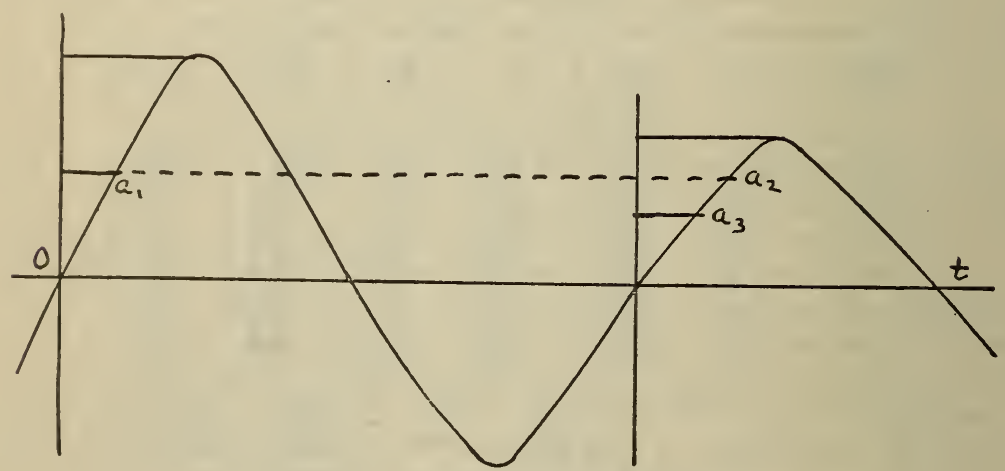

FigURE 14.-Sinusoidal correction

The time of swing in the near position was usually about 1,754 seconds and in the far position about 2,081 seconds, and each could be measured with a precision of about 0.1 second. The difference, 327 seconds, formed the critical quantity of the whole measurement, and may be presumed accurate to about 1 part in 3,300 . It was gratifying to find that the average departure from the mean in each of the two best sets of observations (with the platinum and the glass balls) proved to be of this magnitude.

\section{COMPUTATIONS}

No useful purpose would be served in reproducing here the computations involved in obtaining the results, as these details would be of interest only to some one planning to repeat the work. The complete computations, covering about 1,000 folio sheets, and the original notebooks are preserved at the library of the Bureau of
Standards.

It may, however, be of general interest to see the relative importance of the attraction due to the different moving parts of the apparatus. Table 11 summarizes these attractions for a typical case in the near 
position, and Table 12 does the same for the far position. 'The figures quoted are for one of the experiments with platinum balls. The numbers given represent the contributions of the different moving parts to the quantity $\Sigma(F K)$ in formula (14). The force due to the balls is, of course, the preponderating factor, being about 99 per cent of the whole. The next largest figure is that due to the aluminum rod, less than 1 per cent of the total.

TABLE 11.-Partial values of $\mathrm{z}(F K)$ in the near position

\begin{tabular}{|c|c|c|}
\hline \multicolumn{3}{|c|}{ Attraction of both cylinders on moving parts named } \\
\hline Parts & \multicolumn{2}{|c|}{ Attraction } \\
\hline $\begin{array}{l}\text { Balls } \\
\text { Aluminum rod } \\
\text { Copper truss } \\
\text { Copper in bar } \\
\text { Copper hooks. } \\
\text { Tungsten in moving system } \\
\text { Mirror }\end{array}$ & $\begin{array}{r}419,215 G \\
2,714 G \\
245 G \\
14 G \\
10 G \\
6 G \\
2 G\end{array}$ & \multirow{3}{*}{$422,200 \mathrm{G}$} \\
\hline \multicolumn{2}{|c|}{ Attraction of other fixed parts on balls } & \\
\hline $\begin{array}{l}\text { Supporting rods. } \\
\text { Lower nuts._. } \\
\text { Turnbuckles. } \\
\text { Disks } \\
\text { Ueper nuts... }\end{array}$ & $\begin{array}{l}87 G \\
8 G \\
0 \\
6 G \\
0 \\
5 G\end{array}$ & \\
\hline Total..... & & $422,312 G$ \\
\hline
\end{tabular}

TABLE 12.-Partial values of $\Sigma(F K)$ in the far position

\begin{tabular}{|c|c|c|}
\hline \multicolumn{3}{|c|}{ Attraction of both cylinders on moring parts named } \\
\hline Parts & \multicolumn{2}{|c|}{ Attraction } \\
\hline $\begin{array}{l}\text { Balls } \\
\text { Aluminum rod } \\
\text { Copper truss } \\
\text { Copper in bar } \\
\text { Copper hooks. } \\
\text { Tungsten in moving system. } \\
\text { Mirror }\end{array}$ & $\begin{array}{r}-174,399 G \\
-1,608 G \\
-146 G \\
-6 G \\
-4 G \\
-4 G \\
-2 G\end{array}$ & \multirow{3}{*}{$-176,169 G$} \\
\hline \multicolumn{2}{|c|}{ Attraction of other fixed parts on balls } & \\
\hline $\begin{array}{l}\text { Supporting rods. } \\
\text { Lower nuts } \\
\text { Turnbuckles. } \\
\text { Disks } \\
\text { Upper nuts. } \\
\text { Beam }\end{array}$ & $\begin{array}{c}-44 G \\
-8 G \\
0 \\
-4 G \\
0 \\
-4 G\end{array}$ & \\
\hline Total ................... & & $-176,229 G$ \\
\hline
\end{tabular}




\section{RESULTS}

\section{GOLD BALLS}

It has already been stated in the description of the small masses that trouble was encountered by reason of the gold balls absorbing mercury vapor during the seven months' period occupied by the observations. The total increase in mass of the balls is shown in Table 13.

TABLE 13.-Mass of gold balls in vacuo

\begin{tabular}{|c|c|c|}
\hline Date & Ball A & Ball B \\
\hline $\begin{array}{l}\text { Nov. } 15,1924 \\
\text { June } 15,1925 .\end{array}$ & $\begin{array}{l}48.9688 \\
49.1067\end{array}$ & $\begin{array}{l}\text { 48. } 9847 \\
\text { 49. } 1164\end{array}$ \\
\hline Increase & .1379 & .1317 \\
\hline
\end{tabular}

The actual observations for time of swing were all grouped rather closely together during the last half of this period. The balls were weighed initially on November 15, 1924, and placed in position in the apparatus, which was then closed and exhausted of air. The centering operations required some time and other delays ensued. As a consequence, the first actual measurement of time of swing was made on March 12, 1925. Seven swings were made in all, alternately in the near and the far positions, the final swing occurring on June 8,1925 . The apparatus was opened and the balls reweighed on June 15, 1925.

The average time that elapsed between swings was two weeks. If we assume the absorption of mercury to have proceeded regularly during the whole seven months, the increase in two weeks would be about $0.01 \mathrm{~g}$. for each ball, or about one-twentieth of 1 per cent. If we assume also that the mercury penetrated the gold balls with spherical symmetry (which from the final appearance of the balls seems reasonable) we may interpolate for the mass of the balls at any period of observation without introducing an error of more than perhaps 1 part in 10,000 .

Calculation shows that an error of this magnitude in the mass of the balls will give rise to an error of about 4 parts in 10,000 in the value of $G$. It was, therefore, thought worth while to compute the results for the gold ball set on the basis of the assumptions that have been mentioned. The results are shown in Table 14.

TABLE 14.-Constant of gravitation derived from experiments with gold balls

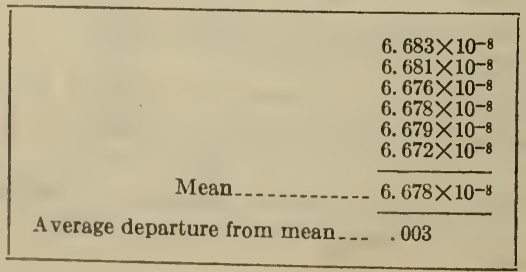

\section{PLATINUM BALLS}

To avoid the difficulty of mercury absorption a second set of measurements was made with platinum balls coated thinly with 
lacquer. The balls were weighed initially on October 10, 1925, and finally on November 17, 1926. The initial weight was that of ball and hook; the final weight included such portion of the tungsten filament as was wrapped around the hook, together with the trace of wax that held it in place. The final weight was used in the computations. The results obtained are shown in Table 15.

TABLE 15.-Masses in vacuo of platinum balls

\begin{tabular}{|c|c|c|c|}
\hline & Date & Ball A & Ball 13 \\
\hline $\begin{array}{l}\text { Oct. } 10,1925 \ldots \\
\text { Nor. } 7,1926 \ldots\end{array}$ & 4. & $\begin{array}{l}50.7116 \\
60.7162\end{array}$ & $\begin{array}{l}50.7271 \\
50.72077\end{array}$ \\
\hline
\end{tabular}

Five values of the constant of gravitation were obtained from this set-up, shown in Table 16.

TABLE 16.-Constant of gravitation derived from experiments wilh platinum balls

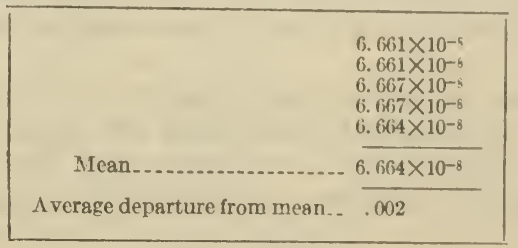

\section{GLASS BALLS}

A third set of experiments was made with balls of optical glass, of density 3.6. These balls, like those of metal, had a mass of approximately $50 \mathrm{~g}$, and, consequently, a diameter of about $3 \mathrm{~cm}$. The balls were made with a finely ground finish and were truly spherical to $0.001 \mathrm{~cm}$.

The masses of the balls are given in Table 17 .

TABLE 17.-Masses of glass balls in vacuo

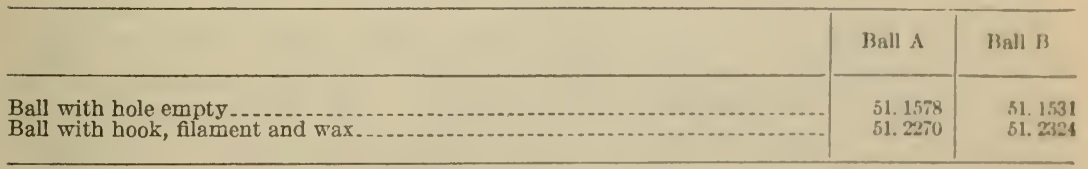

The glass removed in making the hole was in each case about $0.02 \mathrm{~g}$. The final weight, with hook, adhering filament, and wax, was used in the computations.

Five results were obtained with the glass balls, shown in Tahle 18.

TABLE 18.-Constant of gravitation derived from experiments wilh glass balls

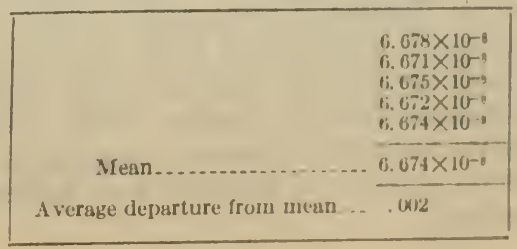


It is interesting to compare these mean results and their measures of precision. This is done in Table 19.

TABLE 19.-Comparison of results

\begin{tabular}{l|r|r|r}
\hline Material & $\begin{array}{c}\text { Extreme } \\
\text { values }\end{array}$ & $\begin{array}{r}\text { Mean } \\
\text { value }\end{array}$ & $\begin{array}{r}\text { A verage } \\
\text { departure } \\
\text { from mean }\end{array}$ \\
\hline Gold._. & & \\
Platinum. & 6.683 \\
6.672 & 6.678 & 0.003 \\
6.667 & 6.661 \\
6.678 & 6.664 & .002 \\
6.671 & 6.674 & .002 \\
\hline
\end{tabular}

\section{MEAN VALUE}

It will be seen that the spread of the gold ball results is about twice as great as those with either platinum or glass, and that there is an overlapping of the spreads with gold and glass. It is evident that the gold ball results are much less reliable than either of the other sets.

The results with platinum and glass may be rated as of equal excellence, the average departure being the same and the spreads small and approximately equal. Discarding the gold results and giving equal weights to platinum and glass we obtain

$$
6.669 \times 10^{-8}
$$

with an average departure from the mean of 0.005 . It seems advisable to us, however, to retain the gold results at one-third the weight of either platinum or glass. This gives as a final result

$$
6.670 \times 10^{-8}
$$

with an average departure from the mean of 0.005 .

\section{DISCUSSION OF RESULTS}

The first thing that attracts attention in the results given in Table 19 is the apparent difference with the nature of the material. The mean values of the platinum and glass sets, for instance, differ by an amount five times as great as the average departure of either set. Moreover, there is no overlap in their spreads.

However, an explanation of this variation on the basis of difference of material is not admissible. As already stated (footnote (1)) Eötvös, by his very ingenious torsion balance method, has demonstrated the constancy of the gravitation constant for a number of materials (including platinum) to a precision of 6 parts in $10^{9}$. He did not, however, test optical glass. While there is no doubt that the result obtained by Eötvös is universally valid, it seemed best to repert his torsion balance experiment with the balls of platinum and glass. This work was carried out by Dr. G. S. Cook, of the Bureau of Standards, and is described in Section VIII. The high precision of the negative results obtained rules out any attempt to explain the variation of results on a basis of difference of material. Some other explanation is necessary. 
To account for the observed differences it is necessary to find some factor which varies in the several series of experiments and does not eliminate in the near and far positions. Perhaps the first explanation that suggests itself is that of incomplete mannetic shielding. The evidence on this point (III, 6) appears conclusive, but is strengthened by the following considerations.

Gold is diamagnetic and platinum paramngnetic. Any residual magnetic effect would, therefore, weaken the attraction ori the gold balls and strengthen it on platinum; but the values of $G$ actually obtained are less for platinum than for gold.

It was recognized that the gravitational field in the room was not uniform, due partly to the upright stand carrying the pump attachments, and partly to the walls of the room. Two of these wero backed with earth and two were merely partitions.

Such a field, however, being stationary, should produce no effect that would not be eliminated in the near and far positions.

It was recognized also that the resting position of the moving system was slightly different with respect to the field of the room in the several series of experiments, but it is not apparent that this would produce any effect that would not be eliminated.

Probably the solution of this difficulty can best bo attained by working with an apparatus sufficiently sensitive to give the next decimal place. Should the variation found fail to appear in its present place, but be shifted farther on, the difficulty, while not explained, would obviously be ascribable to some experimental error.

Such an apparatus has been constructed, in which the difference of the two times of swing is about 100 minutes. This apparatus awaits the construction of the next new building at the Bureau of Standards, when opportunity may. offer to provide a basement room large enough to contain it comfortably.

\section{THE SPECIFIC ATTRACTION OF PLATINUM AND GLASS $^{8}$}

The experimental values of the constant of gravitation as given in the preceding section vary for small masses of different materials by an amount greater than the averag edeparture of the single values for any particular material. It seems reasonable to attribute this to experimental error of some nature, but it is possible to interpret it as a departure from Newton's law of the proportionality of mass and weight.

The Cavendish apparatus is not suited for the detection of rery small departures from this law, since the forces involved are so small, but it is possible to test this point in other ways to a very high degreo of precision. Such experiments have invariably shown, to the degree of precision of which the apparatus was capable, that the ratio of mass to weight is independent of the material. Pendulum experiments conducted by Newton ${ }^{9}$ established this to 1 part in 1,000, and similar experiments by Bessel ${ }^{10}$ of a more refined character car-

8 This section and the experimental work it describes are due to Dr. G. S. Cook, of the Bureau of Stand. ards.

Principia, Book III, Prop. 6, Theorem 6.

10 Pogg. Annalen, 25, p. 401; 1832. 
ried the precision to one part in 60,000 . It is worthy of note that Bessel included among the substances tested by him meteoric iron and meteoric stone.

The most precise experiments are those of Eötvös, ${ }^{11}$ who by an ingenious application of the torsion balance succeeded in pushing the precision to 6 parts in $10^{9}$. The substances examined by him were water, copper, platinum, magnalium, copper sulphate (solid and solution), asbestos and talc. As he did not test glass, it was thought worth while to repeat the Eötvös experiment with one of the glass balls actually employed in the determination of the constant of gravitation balanced against a ball of platinum.

In the Eötvös experiment two masses of different materials are suspended from the ends of the beam of a torsion balance. These masses may be thought of as acted upon by the gravitational attraction of the earth (a force which may not be proportional to the masses of the two bodies), and by a centrifugal force due to the rotation of the earth, which will be proportional to the masses. If then the gravitational force is not proportional to the masses the resultant forces on the two bodies will be in different directions, and in consequence a torque must be supplied by the suspending filament in order to hold the beam in equilibrium in an east and west position. This torque must be reversed if the beam (together with the torsion head) is turned through $180^{\circ}$.

In practice, the whole apparatus, including observing telescope and scale, was rotated. In this way the effect sought would show itself as a change in the equilibrium position.

H. A. Wilson ${ }^{12}$ has given the theory of this experiment in a somewhat simpler form than that employed by Eötvös. The apparatus used in the present work was in dimensions and arrangement similar to that described by Wilson.

The torsion balance consisted of a light aluminum beam about 5 $\mathrm{cm}$ long carrying a glass ball at one end and a platinum ball at the other. The beam was supported at its center by a tungsten filament $0.025 \mathrm{~mm}$ in diameter and about $43 \mathrm{~cm}$ long. The beam was inclosed in a case of cast iron with walls $1 \mathrm{~cm}$ thick to avoid magnetic disturbances, and the case was exhausted of air to a pressure of about 2 $\mathrm{mm}$ of mercury.

To the case was attached a reading telescope and scale such as are used with wall galvanometers. The whole apparatus was placed on a level plate and pivoted so that it might be readily turned about its vertical axis. Deflections were read through a window in the case by means of the telescope and scale. Table 20 shows the readings obtained. 
TABLE 20.-Resting poinls in centimeters obscred with Ë̈leos apparatus

\begin{tabular}{|r|c|}
\hline Platinum ball east & Platinum hall west \\
\hline 1.40 & 1.40 \\
1.35 & 1.37 \\
1.36 & 1.34 \\
1.32 & 1.31 \\
1.27 & 1.27 \\
1.26 & 1.26 \\
1.24 & \\
\hline 1.319 & 1.325 \\
Mean & \\
\hline Scale distance from mirror on beam, $50 \mathrm{~cm}$ \\
\hline
\end{tabular}

The formula given by Wilson is as follows:

$$
\frac{g-g^{\prime}}{g^{\prime}}=(2 \Delta) \frac{2 d t^{2}}{r T^{2} \sin 2 \theta}
$$

in which

$g=$ acceleration of gravitation.

$2 \Delta=$ change in azimuth angle when beam is turned $180^{\circ}$.

$2 d=$ distance between centers of balls suspended from beam.

$t=$ period of the earth's rotation.

$\theta=$ latitude of place of the experiment.

$T=$ period of vibration of the torsion balance.

$r=$ radious of earth.

At Washington $\theta=38^{\circ} 54^{\prime}$, and the following values were observed:

$$
\begin{aligned}
T & =315 \mathrm{sec} . \\
2 d & =4.9 \mathrm{~cm}
\end{aligned}
$$

Taking $g=980$, we get:

$$
\frac{g-g^{\prime}}{g^{\prime}}=2 \Delta \times 6 \times 10^{-4}
$$

In the experiment, $2 \Delta=6 \times 10^{-3}$ consequently

$$
\frac{g-g^{\prime}}{g^{\prime}}=3.6 \times 10^{-8}
$$

While this result, from its minuteness, is of no importance in the present instance, it may be stated for the sake of completeness that the difference, regarded as real, is in the direction which would correspond to the different values of $G$ obtained for glass and platinum. Its only value in the present work is to prove that the difference apparently found with glass and platinum is not to be explained on a basis of difference of material.

The value of $2 \Delta$ obtained from Table 20 is just about at the readable limit, being read from a scale of millimeters. The gradual change 
in resting point shown in Table 20 is the typical behavior of a suspension immediately after a load has been placed upon it. It is quite marked here because of the fact that several hours always elapsed between successive readings. This lapse of time was necessary because of the small damping.

\section{SUMMARY AND GENERAL CONCLUSIONS}

The value adopted for the constant of gravitation as a result of experiments with small masses of gold, platinum, and glass, weighted, respectively, as 1,3 , and 3 , is, in c. g..s. units

$$
6,670 \times 10^{-8} \mathrm{~cm}^{3} \mathrm{~g}^{-1} \mathrm{sec}^{-2}
$$

with a precision, as measured by the average departure from the mean, of 0.005 .

This result agrees well with that obtained by Boys and Braun, namely $6.66 \pm 0.01$.

The different results obtained with the various materials used for the small masses are yet to be explained, but evidence is given that this difference is not to be ascribed to the nature of the material.

Washington, April 10, 1930. 